\title{
Analysis of Coarse-Grained Lattice Models and Connections to Nonlocal Interactions
}

\author{
Qiang $\mathrm{Du}^{1, *}$, Xiantao $\mathrm{Li}^{2}$ and Liming Yuan ${ }^{2}$ \\ 1 Applied Physics and Applied Mathematics, and Data Science Institute, Columbia \\ University, New York, USA. \\ ${ }^{2}$ Department of Mathematics, The Pennsylvania State University, University Park, \\ Pennsylvania, 16802, USA.
}

Received 24 August 2019; Accepted 10 March 2020

\begin{abstract}
We study coarse-grained models of some linear static lattice models with interactions up to second nearest neighbors. It will be demonstrated how nonlocal interactions, as described by a nonlocal kernel function, arise from a coarse-graining procedure. Some important properties of the nonlocal kernels will be established such as its decay rate and positivity. We also study the scaling behavior of the kernel functions as the level of coarse-graining changes. In addition, we suggest closure approximations of the nonlocal interactions that can be expressed in local PDE forms by introducing auxiliary variables.
\end{abstract}

AMS subject classifications: 65N15, 74G15, 70E55

Key words: Linear static models, coarse-graining, next nearest neighbor interactions, nonlocal models.

\section{Introduction}

Coarse-graining of finest scale models has become an important methodology in computer simulations of complex systems in the last two decades. For example, despite rapidly growing computing power, all-atom simulations of materials still remain challenging due to the computational complexity, which has been a major motivation for the development of coarse graining methodology. Reduced models (a.k.a., coarse-grained models) have been shown to be more effective in practice. Starting with a relatively small number of representative degrees of freedom, e.g., local averages, the goal in developing a reduced model is to begin with the many-body atomic interactions and derive a model

*Corresponding author. Email addresses: qd2125@columbia.edu (Q. Du), xli@math.psu.edu (X. Li), lzy104@psu.edu (L. Yuan) 
that only involves the preselected degrees of freedom, see $[13,14,23,24]$ for various perspectives and recent progress.

One remarkable success, in the study of solids with crystal structures, has been the quasi-continuum (QC) method [30]. By assuming the smoothness of the deformation field away from defects and employing a finite-element approximation of the elastic energy, a reduced model is constructed that describes the deformation of pre-selected representative atoms. The key ingredient in such an approach is the Cauchy-Born rule, which obtains the local energy density by considering a unit cell under uniform deformation gradient. Effectively, the reduced model, known as the local quasi-continuum approximation, is expressed in terms of the finite element nodal values.

Most QC type of methods have been motivated by continuum mechanics, and inevitably, they lead to modeling error, which can be quantified when the underlying atomic displacement has certain smoothness, see, for example $[6,18,33]$, the analysis for one-dimensional models. In this paper, however, we will first derive an exact reduced model, with an attempt to understand various properties of the exact interactions among the representative atoms resulting from the coarse-graining process. As an illustration, we consider a one-dimensional lattice system with the interactions being among the first and second neighbors. By assuming that the interaction is linear, we can obtain the exact reduced model explicitly in the form of nonlocal difference equations. Several properties regarding the kernel function that characterizes the coarse-grained interactions can be established. Our first observation is that, in general, this kernel function has no compact support, i.e., the interaction is nonlocal. This observation reflects a strong motivation for the current work, that is, to connect model reduction with nonlocal modeling [7]. It also allows us to draw similarities of the reduced models using nonlocal integral operators to nonlocal peridynamics models, see $[15,29]$. We show that the reduced nonlocal model, through rescaling, has a characteristic interaction range, even though the kernel decays exponentially. The latter observation provides a justification for the finite-ranged interactions that are often used in the parameterization of reduced models [14,23].

Furthermore, we suggest a new approach to approximate the nonlocal continuum formulation of the reduced models, by using a Padé approximation of the dispersion relation. More specifically, the nonlocal operator can be approximated by an integral operator with the kernel function consisting of double exponential functions and delta functions. In the physical domain, these models can be rewritten as higher order PDEs, and we prove the stability of these PDEs. It is our hope that this result sheds some light on the origins and selections of interaction kernel functions in nonlocal continuum models. In particular, this approach associates atomic interactions, in the form of force constants, to the kernel functions in the nonlocal models, and hence leading to kernel functions that are material specific and dependent of the coarse-graining procedure.

The rest of the paper is organized as follows. Section 2 will provide a demonstration of the model reduction procedure of some lattice models, including a model with nearest neighbor interactions, followed by one with next nearest neighbor interactions that leads naturally to nonlocal reduced models. In Section 3, we present a Fourier representation 
of the kernel function. Analysis of properties of the kernel function is illustrated in section 4. Finally, in section 5, we make a connection to nonlocal continuum models with exponential type of kernels.

\section{Lattice models}

In this section, to demonstrate the reduction procedure and investigate the properties of the resulting model, we consider a one-dimensional chain model of a crystal lattice. More specifically, given the equilibrium lattice constant $\varepsilon>0$, we define a one dimensional infinite lattice as follows,

$$
\mathbb{L}=\{x \mid x=n \varepsilon, n \in \mathbb{Z}\} .
$$

We will let $x$ be the undeformed (equilibrium) position of an atom, and $u(x)$ be the displacement of the atom from the equilibrium position.

For typical lattice models used in practice, the lattice spacing $\varepsilon$ is on the order of a few angstroms $\left(10^{-10} \mathrm{~m}\right)$. In order to describe the material deformation on a much larger scale, we define a coarse lattice by selecting a representative atom from each group of $M$ atoms, as done in the quasi-continuum method [30]. To be more specific, the selected representative atoms form a similar lattice structure, i.e.,

$$
\mathbb{L}_{C G}=\{y \mid y=n H, n \in \mathbb{Z}\}, \quad \mathbb{L}_{C G}^{-}=\mathbb{L} \backslash \mathbb{L}_{C G}
$$

where we have set $H=M \varepsilon$, which represents the spatial scale of interest, possibly ranging from nanometer scale $\left(10^{-9} \mathrm{~m}\right)$ to micron scale $\left(10^{-6} \mathrm{~m}\right)$. A pictorial illustration of the two lattices can be found in Fig. 1 . Here $\mathbb{L}_{C G}^{-}$denotes the set of remaining atoms that will be eliminated by the coarse-graining procedure.

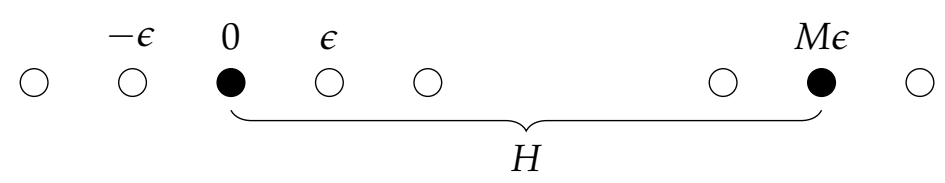

Figure 1: The lattice structure associated with the full atomistic model (open circles), together with the lattice associated with the coarse spatial scale.

\subsection{A lattice model with nearest neighbor interactions}

To illustrate the idea of the model reduction, let us first consider a linear lattice model with nearest neighbor interactions, i.e., each atom is interacting with its immediate neighbors on the one dimensional chain. Let $K>0$ be the elastic constant, and $f \in \ell^{2}(\mathbb{L})$ be an external force function. Then the model can be formulated as a minimization problem, where the quadratic strain energy is given by

$$
E[u]=\sum_{x \in \mathbb{L}} \frac{K}{2}\left(\frac{u(x+\varepsilon)-u(x)}{\varepsilon}\right)^{2}-f(x) u(x) .
$$


One can use the variational principle for the energy functional $E$ in (2.3) and obtain the Euler-Lagrange equation, which yields a force balance equation,

$$
L u:=-K \frac{u(x+\varepsilon)-2 u(x)+u(x-\varepsilon)}{\varepsilon^{2}}=f(x) .
$$

Starting with the lattice model (2.4), we turn to the deformation of the representative atoms on the coarse scale $H$. In particular, we aim to reduce the degrees of freedom by deriving a new equation that accounts only for the displacement of the representative atoms. One simple approach to perform the reduction procedure is to use the lattice Green's function $g\left(x, x^{\prime}\right)$, which is defined on $\mathbb{L} \times \mathbb{L}$ by

$$
\operatorname{Lg}\left(x, x^{\prime}\right)=\varepsilon^{-1} \delta\left(x-x^{\prime}\right),
$$

where

$$
\delta(x)= \begin{cases}1, & x=0, \\ 0, & \text { otherwise, }\end{cases}
$$

is the Kronecker delta function. See for example [19]. In fact, the Green's function for this one-dimensional lattice model has a closed form, given by

$$
g\left(x, x^{\prime}\right)=-\frac{\left|x-x^{\prime}\right|}{2 K}, \quad \forall x, x^{\prime} \in \mathbb{L} .
$$

To move forward, we also need Green's identities (Lemma A.2) that, in the discrete setting, amount to summation by parts (Lemma A.1). Details are provided in the appendices.

With all the ingredients ready, the original model on the full lattice $\mathbb{L}$ can be reduced to a model on $\mathbb{L}_{C G}$. This can be understood in an intuitive way: Since we want to keep the representative atoms, other atoms can be eliminated by solving boundary value problems associated with the original lattice model over a cell between two neighboring atoms on the coarse lattice $\mathbb{L}_{C G}$. Simply multiplying Eq. (2.4) by the Green's function, then applying the Green's identity, we obtain an equation on $\mathbb{L}_{C G}$ for the displacement of the representative atoms,

$$
-K \frac{u(y+H)-2 u(y)+u(y-H)}{H^{2}}=\frac{\varepsilon}{H} \sum_{\substack{x \in \mathbb{L} \\ y-H<x<y+H}} h(x, y) f(x), \quad \forall y \in \mathbb{L}_{C G},
$$

where $h(x, y)$ is a nonnegative function taking value 1 at $x=y$ and 0 at $x=y+H, y-H$. For details of the derivation, see the Appendices.

The main observation here is that the coarse-scale model is still local, though this is a property that is only associated with this one-dimensional model with nearest neighbor interactions. In the next section, we will show that this property breaks down for lattice models with next nearest neighbor interactions. Indeed, the atomic degrees of freedom in general can not be eliminated without involving all the representative atoms. 


\subsection{A lattice model with next nearest neighbor interactions}

Next, we consider a lattice model involving next nearest neighbor interactions, where each atom interacts with two neighboring atoms on each side. The corresponding total energy takes the form of

$$
E[u]=\sum_{x \in \mathbb{L}} \frac{K_{1}}{2}\left(\frac{u(x+\varepsilon)-u(x)}{\varepsilon}\right)^{2}+\frac{K_{2}}{2}\left(\frac{u(x+2 \varepsilon)-u(x)}{\varepsilon}\right)^{2}-f(x) u(x) .
$$

Here $K_{1}$ and $K_{2}$ are the force constants, and we assume that the phonon stability condition is satisfied [12]:

$$
K_{1}>0, \quad K_{1}+4 K_{2}>0 .
$$

The variational principle leads to the equation,

$$
L u:=-K_{1} \frac{u(x+\varepsilon)-2 u(x)+u(x-\varepsilon)}{\varepsilon^{2}}-K_{2} \frac{u(x+2 \varepsilon)-2 u(x)+u(x-2 \varepsilon)}{\varepsilon^{2}}=f(x) .
$$

This is a difference equation defined on $\mathbb{L}$ that can be viewed as a discretization of the Poisson equation,

$$
-\left(K_{1}+4 K_{2}\right) u^{\prime \prime}(x)=f(x),
$$

which can be regarded as a one-dimensional elasticity model with elastic constant $K:=$ $K_{1}+4 K_{2}$.

Again, the linear operator $L$ admits a Green's function, which takes the form of

$$
g\left(x-x^{\prime}\right)=\alpha\left|x-x^{\prime}\right|+\beta z^{\left|x-x^{\prime}\right| / \varepsilon}-\beta, \quad \forall x, x^{\prime} \in \mathbb{L},
$$

with parameters $\alpha, \beta$ and $z$ obtained from $K_{1}$ and $K_{2}$.

Following the same procedure as before, we can remove the non-representative atoms $\mathbb{L}_{C G}^{-}$, and derive a set of equations only involving the displacement of the representative atoms on $\mathbb{L}_{C G}$. The reduced model can be written as

$$
\sum_{y^{\prime} \in \mathbb{L}_{C G}} \theta\left(y-y^{\prime}\right) u\left(y^{\prime}\right)=\bar{f}(y), \quad \forall y \in \mathbb{L}_{C G} .
$$

Here $\bar{f}(x)$ is defined in terms of the body force $f(x)$ and the Green's function (2.13). This procedure is similar to the Schur complement reduction or the boundary element formulation [17]. Due to the lengthy derivation, we have left the details in the Appendices.

The function $\theta$ will be referred to as the nonlocal kernel function (see Theorem 3.2). The main observation here is that the reduced model on the coarse lattice $\mathbb{L}_{C G}$ is nonlocal: the displacement of all the representative atoms are in principle coupled with every other representative atom. The rest of this paper is concerned with the properties and approximations of this kernel function. 


\section{Nonlocal interactions via the Fourier transform}

In this section, we reformulate the reduced model (2.14) and study the nonlocal interaction kernel using Fourier transform. This approach reveals a more intuitive coarsegraining process through direct eliminations, and it also offers an effective tool for more detailed analysis.

In light of the symmetry and translation invariance of Eq. (2.11), for functions $u \in$ $\ell^{2}(\mathbb{L})$ and $f \in \ell^{2}(\mathbb{L})$, one may take the Fourier transform in a block-wise manner to arrive at a finite-dimensional matrix representation of the reduction procedure. In particular, it leads to the Fourier transform of $\theta$.

\subsection{Operator formulation and function spaces}

We begin with $\ell^{2}(\mathbb{L})$, the Hilbert space of square integrable functions defined on the lattice $\mathbb{L}$,

$$
\ell^{2}(\mathbb{L})=\left\{u(x) \mid \sum_{x \in \mathbb{L}} u^{2}(x)<\infty\right\}
$$

Similarly we define $\ell^{2}\left(\mathbb{L}_{C G}\right)$ and $\ell^{2}\left(\mathbb{L}_{C G}^{-}\right)$for $\mathbb{L}_{C G}$ and $\mathbb{L}_{C G}^{-}$defined in (2.2).

Recall that to obtain the coarse lattice, we simply select one representative atom for each group of consecutive $M$ atoms. This selection procedure can be expressed in terms of the following linear mappings:

$$
\begin{array}{lll}
\Phi: & \ell^{2}(\mathbb{L}) \rightarrow \ell^{2}\left(\mathbb{L}_{C G}\right), & u(x) \mapsto v(x), \\
\Psi: & \ell^{2}(\mathbb{L}) \rightarrow \ell^{2}\left(\mathbb{L}_{C G}^{-}\right), & u(x) \mapsto w(x) .
\end{array}
$$

Note that the setup requires $v(x)=\Phi u(x)$ and $w(x)=\Psi u(x)$ satisfying $v(x)=u(x), \forall x \in$ $\mathbb{L}_{C G}$ and $w(x)=u(x), \forall x \in \mathbb{L}_{C G}^{-}$.

With those Hilbert spaces set up, the adjoint operators are given via duality respectively

$$
\begin{aligned}
& (\Phi u, s)_{\ell^{2}\left(\mathbb{L}_{C G}\right)}=\left(u, \Phi^{*} s\right)_{\ell^{2}(\mathbb{L})^{\prime}} \\
& (\Psi u, t)_{\ell^{2}\left(\mathbb{L}_{C G}^{-}\right)}=\left(u, \Psi^{*} t\right)_{\ell^{2}(\mathbb{L})^{\prime}}
\end{aligned}
$$

for all $s \in \ell^{2}\left(\mathbb{L}_{C G}\right)$ and $t \in \ell^{2}\left(\mathbb{L}_{C G}^{-}\right)$. One may view $\Phi$ and $\Psi$ as the restriction operators as in conventional multigrid/multiscale analysis [21]. The way that the two operators $\Phi$ and $\Psi$ are defined implies that $\Phi^{*}$ and $\Psi^{*}$ have range supported in $\mathbb{L}_{C G}$ and $\mathbb{L}_{C G}^{-}$, respectively. For example, $\Phi^{*} s$ would be a function in $\ell^{2}(\mathbb{L})$ with zero values away from the representative atoms. Therefore for any $u \in \ell^{2}(\mathbb{L})$, there exist some $v \in \ell^{2}\left(\mathbb{L}_{C G}\right)$ and $w \in \ell^{2}\left(\mathbb{L}_{C G}^{-}\right)$, such that $u=\Phi^{*} v+\Psi^{*} w$, a fact that we will use later. 
Next we explore the operator $L: \ell^{2}(\mathbb{L}) \rightarrow \ell^{2}(\mathbb{L})$ as in Eq. (2.11). It is intuitive to think of $L$ as the following infinite matrix

$$
L=\left[\begin{array}{ccccccc} 
& \vdots & \vdots & \vdots & & & \\
\cdots & -K_{1} & 2 K_{1}+2 K_{2} & -K_{1} & -K_{2} & \cdots & \\
& -K_{2} & -K_{1} & 2 K_{1}+2 K_{2} & -K_{1} & -K_{2} & \\
& & -K_{2} & -K_{1} & 2 K_{1}+2 K_{2} & -K_{1} & -K_{2} \\
& & & \vdots & \vdots & \vdots &
\end{array}\right]
$$

By definition, such an operator $L$ is a Toeplitz operator generated by the vector

$$
\left(\cdots, 0,-K_{2},-K_{1}, 2 K_{1}+2 K_{2},-K_{1},-K_{2}, 0, \cdots\right) .
$$

Theorem 1.1 in [2] states that $L$ is a bounded self-adjoint operator on $\ell^{2}(\mathbb{L})$.

Naturally, we can also consider a mapping for the reduced model (2.14),

$$
\Theta: \ell^{2}\left(\mathbb{L}_{C G}\right) \rightarrow \ell^{2}\left(\mathbb{L}_{C G}\right) .
$$

In Theorem 3.1, we shall derive the reduced equation (2.14) in terms of operators, where the precise formula of $\Theta$ is given.

Theorem 3.1. Given $M \in \mathbb{N}$ and $M>5, \Theta$ is an operator given by

$$
\Theta=\left(\Phi \circ L \circ \Phi^{*}\right)-\left(\Phi \circ L \circ \Psi^{*}\right)\left(\Psi \circ L \circ \Psi^{*}\right)^{-1}\left(\Psi \circ L \circ \Phi^{*}\right) .
$$

Proof. We first need the fact that $\Psi \circ L \circ \Psi^{*}$ is invertible, which will be proved in Lemma 3.2.

Now consider the equation $L u=f$ for $u, f \in \ell^{2}(\mathbb{L})$. Let us define as above $v=\Phi u$ and $w=\Psi u$ so that $v \in \ell^{2}\left(\mathbb{L}_{C G}\right)$ and $w \in \ell^{2}\left(\mathbb{L}_{C G}^{-}\right)$. For $\forall s \in \ell^{2}(\mathbb{L})$, we have

$$
\begin{aligned}
\left(\Phi^{*} v, s\right)_{\ell^{2}(\mathbb{L})}+\left(\Phi^{*} w, s\right)_{\ell^{2}(\mathbb{L})} & =(v, \Phi s)_{\ell^{2}\left(\mathbb{L}_{C G}\right)}+(w, \Psi s)_{\ell^{2}\left(\mathbb{L}_{C G}^{-}\right)} \\
& =(\Phi u, \Phi s)_{\ell^{2}\left(\mathbb{L}_{C G}\right)}+(\Psi u, \Psi s)_{\ell^{2}\left(\mathbb{L}_{C G}^{-}\right)} \\
& =(u, s)_{\ell^{2}(\mathbb{L})} .
\end{aligned}
$$

The equality holds since $\Phi$ and $\Psi$ are projections and inner products are summable. It is essentially a change of order in the summation for $\ell^{2}(\mathbb{L})$ innerproduct, which is valid here because the summation is bounded due to Cauchy-Schwarz inequality. Therefore we have $u=\Phi^{*} v+\Psi^{*} w$.

Operating $\Phi$ and $\Psi$ on this equation, we get

$$
\left\{\begin{array}{l}
\left(\Phi \circ L \circ \Phi^{*}\right) v+\left(\Phi \circ L \circ \Psi^{*}\right) w=\Phi f \\
\left(\Psi \circ L \circ \Phi^{*}\right) v+\left(\Psi \circ L \circ \Psi^{*}\right) w=\Psi f,
\end{array}\right.
$$


for some $v \in \ell^{2}\left(\mathbb{L}_{C G}\right)$ and $w \in \ell^{2}\left(\mathbb{L}_{C G}^{-}\right)$, such that $u=\Phi^{*} v+\Psi^{*} w$.

To derive an equation for functions on $\mathbb{L}_{C G}$, we simply eliminate $w$ from the second equation in (3.7) by making use of the invertibility of $\Psi \circ L \circ \Psi^{*}$. This yields

$$
\begin{aligned}
& {\left[\left(\Phi \circ L \circ \Phi^{*}\right)-\left(\Phi \circ L \circ \Psi^{*}\right)\left(\Psi \circ L \circ \Psi^{*}\right)^{-1}\left(\Psi \circ L \circ \Phi^{*}\right)\right] v } \\
= & \Phi f-\left(\Phi \circ L \circ \Psi^{*}\right)\left(\Psi \circ L \circ \Psi^{*}\right)^{-1} \circ \Psi f .
\end{aligned}
$$

Comparing (3.4) with (3.8), we obtain the operator $\Theta$ and $\bar{f}$.

Remark 3.1. The operator formulation takes an explicit closed form, which is helpful to explore the mathematical properties of $\Theta$. However, when it comes to the numerical implementations, the term $\Psi \circ L \circ \Psi^{*}$ does not offer a more competitive approach than some others such as the one using the Green's functions $[17,34]$.

\subsection{Blockwise Fourier transform}

Here we introduce the notation of the blockwise Fourier transform. Plancherel's Theorem states that each element in $\ell^{2}(\mathbb{L})$ is uniquely associated with a function in $L^{2}(\mathbb{T})$ by Fourier transform, where $\mathbb{T}$ is the torus. We borrow this idea to examine the operator $\Theta$.

We note first that the operator $\Theta$ is defined only for functions in $\ell^{2}\left(\mathbb{L}_{C G}\right)$. To address this issue, we start by taking Fourier transform of $L$ in a block form, in that every $M$ atoms in each block is viewed as one point in $\mathbb{L}_{C G}$. From the lattice structures, this amounts to a complex lattice with each unit cell containing $M$ atoms. More precisely, for each $u(x) \in \ell^{2}(\mathbb{L})$, we can define a vector-valued function $\tilde{u}(x), \forall x \in \mathbb{L}_{C G}$, such that

$$
\tilde{u}(x)=(u(x), u(x+\varepsilon), \cdots, u(x+(M-1) \varepsilon))^{*} .
$$

Therefore, the Fourier transform is a one-to-one mapping from $\ell^{2}\left(\mathbb{L}_{C G}\right)^{M}$ to $L^{2}(\mathbb{T})^{M}$. Similarly, in order to define the Fourier transform on $\mathbb{L}_{C G}^{-}$, we can define for each $u(x) \in$ $\mathbb{L}_{C G}^{-}$a vector-valued function $\bar{u}(x), \forall x \in \mathbb{L}_{C G}$, such that

$$
\bar{u}(x)=(u(x+\varepsilon), \cdots, u(x+(M-1) \varepsilon))^{*} .
$$

Therefore, the Fourier transform is a one-to-one mapping from $\ell^{2}\left(\mathbb{L}_{C G}\right)^{M-1}$ to $L^{2}(\mathbb{T})^{M-1}$.

To proceed, we define the following matrices:

$$
\phi=\left[\begin{array}{llll}
1 & 0 & \cdots & 0
\end{array}\right]_{1 \times M^{\prime}} \quad \psi=\left[\begin{array}{cccc}
0 & 1 & 0 & \cdots \\
0 & 0 & 1 & \cdots \\
\vdots & \vdots & \vdots &
\end{array}\right]_{(M-1) \times M} .
$$

If we represent the operators $\Phi$ and $\Psi$ with infinite matrices and use $\phi$ and $\psi$ as blocks, then $\Phi$ and $\Psi$ are Toeplitz operators generated by $(\cdots, 0, \phi, 0, \cdots)$ and $(\cdots, 0, \psi, 0, \cdots)$, respectively. Based on this observation, we can take the Fourier transform, yielding

$$
\mathcal{F}(\Phi)=\phi, \quad \mathcal{F}(\Psi)=\psi
$$


Notice that both Fourier transforms are constant matrices that are independent of the Fourier variable $\xi$, due to the fact that operators $\Phi$ and $\Psi$ are block diagonal as infinite matrices.

In the same manner, we view $L$ as being generated by $\left(\cdots, 0, L_{1}, L_{0}, L_{1}^{*}, 0, \cdots\right)$ so that

$$
\mathcal{F}(L)=L_{0}+L_{1} e^{-i \xi H}+L_{1}^{*} e^{i \xi H},
$$

where $H$ is the spacing parameter of $\mathbb{L}_{C G}, \xi \in(-\pi / H, \pi / H)$ and

$$
\begin{aligned}
& L_{0}=\varepsilon^{-2}\left[\begin{array}{ccccccc}
2 K_{1}+2 K_{2} & -K_{1} & -K_{2} & 0 & 0 & \cdots & 0 \\
-K_{1} & 2 K_{1}+2 K_{2} & -K_{1} & -K_{2} & 0 & \cdots & 0 \\
-K_{2} & -K_{1} & 2 K_{1}+2 K_{2} & -K_{1} & -K_{2} & \cdots & 0 \\
\vdots & \vdots & \vdots & \vdots & \vdots & \vdots & \vdots
\end{array}\right]_{M \times M}, \\
& L_{1}=\varepsilon^{-2}\left[\begin{array}{cccc}
0 & \cdots & -K_{2} & -K_{1} \\
0 & \cdots & 0 & -K_{2} \\
\vdots & \vdots & \vdots & \vdots \\
0 & \cdots & 0 & 0
\end{array}\right]_{M \times M} .
\end{aligned}
$$

To facilitate the necessary computations, it is helpful to observe the following properties.

Lemma 3.1. Suppose $S$ and $T$ are Toeplitz operators such that the composition $S \circ T$ is welldefined, and $\mathcal{F}$ is the Fourier transform, then

1. $\mathcal{F}(S \circ T)=\mathcal{F}(S) \mathcal{F}(T)$. In particular, $\mathcal{F}\left(S^{-1}\right)=\mathcal{F}(S)^{-1}$ if $S$ is invertible.

2. $\mathcal{F}\left(S^{*}\right)=\mathcal{F}(S)^{*}$.

Proof. The proof depends on the one-to-one correspondence of $\ell^{2}$ and the $L^{2}$ space as explained at the beginning, which allows us to take Fourier transform. Now the composition of Toeplitz operators $S$ and $T$ can be expanded as a convolution, and under the assumption that $S \circ T$ is well-defined, Fourier transform turns convolution into products. The rest is immediate by definition.

Utilizing the above setup, we can prove the invertibility of $\Psi \circ L \circ \Psi^{*}$.

Lemma 3.2. For any $M>5, \Phi \circ L \circ \Phi^{*}$ and $\Psi \circ L \circ \Psi^{*}$ are invertible.

Proof. In our setting, $\Phi \circ L \circ \Phi^{*}=\varepsilon^{-2}\left(2 K_{1}+2 K_{2}\right) i d$ where $i d$ is the identity operator from $\mathbb{L}_{C G}$ onto itself, therefore invertible. On the other hand, we notice that $\Psi \circ L \circ \Psi^{*}$ has the 
following form,

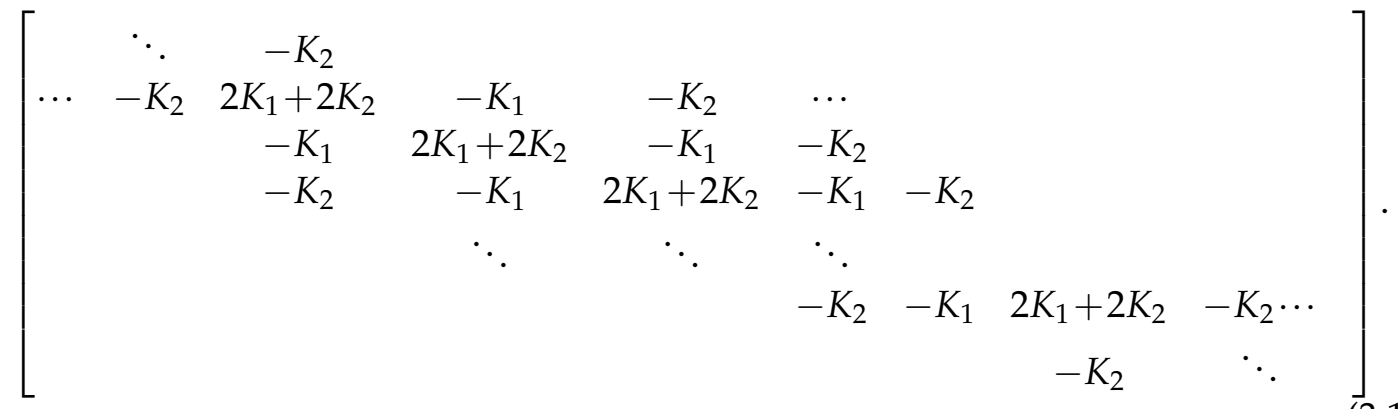

Its Fourier transform on $\ell^{2}\left(\mathbb{L}_{C G}^{-}\right)$, according to our block-wise definition, is given by

$$
\mathcal{F}\left(\Psi \circ L \circ \Psi^{*}\right)=\psi_{0}+\psi_{1} e^{-i \xi^{\tau} H}+\psi_{1}^{*} e^{i \xi^{*} H},
$$

where

$$
\psi_{0}=\varepsilon^{-2}\left[\begin{array}{ccccccc}
2 K_{1}+2 K_{2} & -K_{1} & -K_{2} & 0 & 0 & \cdots & 0 \\
-K_{1} & 2 K_{1}+2 K_{2} & -K_{1} & -K_{2} & 0 & \cdots & 0 \\
-K_{2} & -K_{1} & 2 K_{1}+2 K_{2} & -K_{1} & -K_{2} & \cdots & 0 \\
\vdots & \vdots & \vdots & \vdots & \vdots & \vdots & \vdots
\end{array}\right]_{(M-1) \times(M-1)}
$$

and

$$
\psi_{1}=\varepsilon^{-2}\left[\begin{array}{ccc}
0 & \cdots & -K_{2} \\
\vdots & \vdots & \vdots \\
0 & \cdots & 0
\end{array}\right]_{(M-1) \times(M-1)}
$$

We now show $\mathcal{F}\left(\Psi \circ L \circ \Psi^{*}\right)$ is positive definite. For $X=\left(x_{1}, \ldots, x_{M-1}\right)^{*} \in \mathbb{C}^{(M-1) \times 1}$, we have

$$
\begin{aligned}
& X^{*}\left(\psi \mathcal{F}(L) \psi^{*}\right) X \\
& =\sum_{j=1}^{M-1}\left(2 K_{1}+2 K_{2}\right) \varepsilon^{-2}\left|x_{j}\right|^{2}-\sum_{j=1}^{M-2} K_{1} \varepsilon^{-2}\left(\bar{x}_{j} x_{j+1}+\bar{x}_{j+1} x_{j}\right) \\
& -\sum_{j=1}^{M-3} K_{2} \varepsilon^{-2}\left(\bar{x}_{j} x_{j+2}+\bar{x}_{j+2} x_{j}\right)-K_{2} \varepsilon^{-2} e^{-i \xi} \bar{x}_{1} x_{M-1}-K_{2} \varepsilon^{-2} e^{i \xi^{\tau} H} \bar{x}_{M-1} x_{1} \\
& =\sum_{j=1}^{M-2} K_{1} \varepsilon^{-2}\left|x_{j}-x_{j+1}\right|^{2}+\sum_{j=1}^{M-3} K_{2} \varepsilon^{-2}\left|x_{j}-x_{j+2}\right|^{2}+K_{1} \varepsilon^{-2}\left|x_{1}\right|^{2}+K_{1} \varepsilon^{-2}\left|x_{M-1}\right|^{2} \\
& +K_{2} \varepsilon^{-2}\left|x_{1}\right|^{2}+K_{2} \varepsilon^{-2}\left|x_{2}\right|^{2}+K_{2} \varepsilon^{-2}\left|x_{M-2}\right|^{2}+K_{2} \varepsilon^{-2}\left|x_{M-1}\right|^{2} \\
& -K_{2} \varepsilon^{-2} e^{-i \xi H} \bar{x}_{1} x_{M-1}-K_{2} \varepsilon^{-2} e^{i \xi H} \bar{x}_{M-1} x_{1} \text {. }
\end{aligned}
$$


Here we notice that

$$
\left|x_{j}-x_{j+2}\right|^{2}=\left|x_{j}-x_{j+1}+x_{j+1}-x_{j+2}\right|^{2} \leq 2\left(\left|x_{j}-x_{j+1}\right|^{2}+\left|x_{j+1}-x_{j+2}\right|^{2}\right),
$$

and by rearranging the terms, we arrive at

$$
\begin{aligned}
& X^{*}\left(\psi \mathcal{F}(L) \psi^{*}\right) X \\
& \geq \sum_{j=2}^{M-3}\left(K_{1}+4 K_{2}\right) \varepsilon^{-2}\left|x_{j}-x_{j+1}\right|^{2}+\left(2 K_{1}+3 K_{2}\right) \varepsilon^{-2}\left|x_{1}\right|^{2}+\left(K_{1}+3 K_{2}\right) \varepsilon^{-2}\left|x_{2}\right|^{2} \\
& +\left(K_{1}+3 K_{2}\right) \varepsilon^{-2}\left|x_{M-2}\right|^{2}+\left(2 K_{1}+3 K_{2}\right) \varepsilon^{-2}\left|x_{M-1}\right|^{2}-\left(K_{1}+2 K_{2}\right) \varepsilon^{-2}\left(\bar{x}_{1} x_{2}+\bar{x}_{2} x_{1}\right) \\
& -\left(K_{1}+2 K_{2}\right) \varepsilon^{-2}\left(\bar{x}_{M-1} x_{M-2}+\bar{x}_{M-2} x_{M-1}\right)-K_{2} \varepsilon^{-2}\left(e^{-i \xi H} \bar{x}_{1} x_{M-1}+e^{i \xi H} \bar{x}_{M-1} x_{1}\right) \\
& \geq \sum_{j=2}^{M-3}\left(K_{1}+4 K_{2}\right) \varepsilon^{-2}\left|x_{j}-x_{j+1}\right|^{2}+\left(K_{1}+2 K_{2}\right) \varepsilon^{-2}\left|\sqrt{\frac{K_{1}+2 K_{2}}{K_{1}+3 K_{2}}} x_{1}-\sqrt{\frac{K_{1}+3 K_{2}}{K_{1}+2 K_{2}}} x_{2}\right|^{2} \\
& +\left(K_{1}+2 K_{2}\right) \varepsilon^{-2}\left|\sqrt{\frac{K_{1}+2 K_{2}}{K_{1}+3 K_{2}}} x_{M-1}-\sqrt{\frac{K_{1}+3 K_{2}}{K_{1}+2 K_{2}}} x_{M-2}\right|^{2} \\
& +\frac{K_{1}^{2}+5 K_{1} K_{2}+5 K_{2}^{2}}{K_{1}+3 K_{2}} \varepsilon^{-2}\left|x_{1}-\frac{K_{2}\left(K_{1}+3 K_{2}\right)}{K_{1}^{2}+5 K_{1} K_{2}+5 K_{2}^{2}} e^{-i \xi H} x_{M-1}\right|^{2} \\
& +\varepsilon^{-2}\left(1-\frac{K_{2}^{2}\left(K_{1}+3 K_{2}\right)^{2}}{\left(K_{1}^{2}+5 K_{1} K_{2}+5 K_{2}^{2}\right)^{2}}\right)\left|x_{M-1}\right|^{2} \geq 0 .
\end{aligned}
$$

The last inequality is due to the stability assumption 2.10. Notice that the equality can not hold unless $X=0$. Therefore, the inverse operator, according to Lemma 3.1, is given by the inverse Fourier transform of $\left[\mathcal{F}\left(\Psi \circ L \circ \Psi^{*}\right)\right]^{-1}$.

Now we state the main theorem regarding Fourier transform of the operator $\Theta$.

Theorem 3.2. Let $\mathcal{F}$ be the Fourier transform defined as above and $\xi \in \mathbb{R} \backslash\{0\}$, then

$$
\hat{\theta}(\xi)=\left(\phi \mathcal{F}(L)^{-1} \phi^{*}\right)^{-1} \text {. }
$$

Proof. We shall take Fourier transform and adopt the matrix notation throughout the proof. By definition,

$$
\left[\begin{array}{l}
\phi \\
\psi
\end{array}\right]\left[\begin{array}{ll}
\phi^{*} & \psi^{*}
\end{array}\right]=I .
$$

Therefore, with direct multiplications, we get

$$
\left[\begin{array}{ll}
\phi \mathcal{F}(L) \phi^{*} & \phi \mathcal{F}(L) \psi^{*} \\
\psi \mathcal{F}(L) \phi^{*} & \psi \mathcal{F}(L) \psi^{*}
\end{array}\right]^{-1}=\left[\begin{array}{ll}
\phi \mathcal{F}(L)^{-1} \phi^{*} & \phi \mathcal{F}(L)^{-1} \psi^{*} \\
\psi \mathcal{F}(L)^{-1} \phi^{*} & \psi \mathcal{F}(L)^{-1} \psi^{*}
\end{array}\right]
$$


Now Lemma 3.2 states that $\psi \mathcal{F}(L) \psi^{*}$ is invertible, we can then take the Schur complement and find the inverse,

$$
\left(\psi \mathcal{F}(L) \psi^{*}\right)^{-1}=\psi \mathcal{F}(L)^{-1} \psi^{*}-\psi \mathcal{F}(L)^{-1} \phi^{*}\left(\phi \mathcal{F}(L)^{-1} \phi^{*}\right)^{-1} \phi \mathcal{F}(L)^{-1} \psi^{*}
$$

Notice that $\phi \mathcal{F}(L) \phi^{*}=\left(2 K_{1}+2 K_{2}\right) \varepsilon^{-2}$ and $\psi^{*} \psi=I-\phi^{*} \phi$. Collecting terms, we get from Eq. (3.8) that

$$
\begin{aligned}
\hat{\theta}(\xi)= & \phi \mathcal{F}(L) \phi^{*}-\phi \mathcal{F}(L) \psi^{*}\left(\psi \mathcal{F}(L) \psi^{*}\right)^{-1} \psi \mathcal{F}(L) \phi^{*} \\
= & \left(2 K_{1}+2 K_{2}\right) \varepsilon^{-2}-\phi \mathcal{F}(L) \psi^{*}\left[\psi \mathcal{F}(L)^{-1} \psi^{*}\right. \\
& \left.-\psi \mathcal{F}(L)^{-1} \phi^{*}\left(\phi \mathcal{F}(L)^{-1} \phi^{*}\right)^{-1} \phi \mathcal{F}(L)^{-1} \psi^{*}\right] \psi \mathcal{F}(L) \phi^{*} \\
= & \left(2 K_{1}+2 K_{2}\right) \varepsilon^{-2}-\phi \mathcal{F}(L) \psi^{*} \psi \mathcal{F}(L)^{-1} \psi^{*} \psi \mathcal{F}(L) \phi^{*} \\
& \quad-\phi \mathcal{F}(L) \psi^{*} \psi \mathcal{F}(L)^{-1} \phi^{*}\left(\phi \mathcal{F}(L)^{-1} \phi^{*}\right)^{-1} \phi \mathcal{F}(L)^{-1} \psi^{*} \psi \mathcal{F}(L) \phi^{*} \\
= & \left(2 K_{1}+2 K_{2}\right) \varepsilon^{-2}-\phi \mathcal{F}(L)\left(I-\phi^{*} \phi\right) \mathcal{F}(L)^{-1}\left(I-\phi^{*} \phi\right) \mathcal{F}(L) \phi^{*} \\
& \quad-\phi \mathcal{F}(L)\left(I-\phi^{*} \phi\right) \mathcal{F}(L)^{-1} \phi^{*}\left(\phi \mathcal{F}(L)^{-1} \phi^{*}\right)^{-1} \phi \mathcal{F}(L)^{-1}\left(I-\phi^{*} \phi\right) \mathcal{F}(L) \phi^{*} \\
= & 2\left(2 K_{1}+2 K_{2}\right) \mathcal{E}^{-2}-\left(2 K_{1}+2 K_{2}\right)^{2} \mathcal{E}^{-4} \phi \mathcal{F}(L)^{-1} \phi^{*} \\
& \quad+\left(\phi \mathcal{F}(L)^{-1} \phi^{*}\right)^{-1}-2\left(2 K_{1}+2 K_{2}\right) \mathcal{E}^{-2}+\left(2 K_{1}+2 K_{2}\right)^{2} \varepsilon^{-4} \phi \mathcal{F}(L)^{-1} \phi^{*} \\
= & \left(\phi \mathcal{F}(L)^{-1} \phi^{*}\right)^{-1} .
\end{aligned}
$$

The proof is completed.

This procedure can be extended to more general settings involving other choices of operators $\Phi$ and $\Psi$, e.g., those considered in $[3,16]$.

\section{Properties of nonlocal operator}

This section is devoted to quantitative analysis of properties of the nonlocal operator $\Theta$. The reduced model depends entirely on the operator $\Theta$, so it makes sense to see what kind of properties are inherited by the reduced model by exploring $\Theta$. Relevant results are summarized in Theorem 4.1.

Meanwhile, the reduced model exhibits nonlocal interactions spreading over the whole domain. In practical applications, it is important to understand how strong the pairwise interactions are in the coarse-grained model. Theorem 4.2 suggests that such nonlocal interactions through the coarse-graining performed in the previous section decay faster than any algebraic power. Numerical simulations show that the decay is exponential. See Fig. 3.

In the case where $K_{2}>0$, given a finite lattice with proper boundary conditions, Eq. (2.11) is well posed and discrete maximum principle applies, see [4]. Similarly for the reduced model, the maximum principle like properties are inherited by $\theta$ (the nonlocal kernel function defined in Theorem 4.1), as stated in Theorem 4.3 and 4.4. 


\subsection{Some basic facts}

First we list basic facts about $\Theta$ in the following theorem.

Theorem 4.1. The nonlocal operator $\Theta$ satisfies:

1. $\Theta$ is translation invariant, i.e. there exist $\theta \in \ell^{2}\left(\mathbb{L}_{C G}\right)$ such that

$$
\Theta u=\sum_{y^{\prime} \in \mathbb{L}_{C G}} \Theta\left(y, y^{\prime}\right) u\left(y^{\prime}\right)=\sum_{y^{\prime} \in \mathbb{L}_{C G}} \theta\left(y-y^{\prime}\right) u\left(y^{\prime}\right) .
$$

We shall call $\theta$ the nonlocal kernel function.

2. $\theta$ is an even function and has a zero sum. Consequently, $\hat{\theta}(0)=0$ in Theorem 3.2.

Proof. To prove (1), we first examine the coarse-graining procedure. The full lattice and the linear operator $L$ are symmetric about 0 and translation invariant, i.e.,

$$
L[u(-x)]=[L u](-x), \quad L[u(x+t)]=[L u](x+t) .
$$

If we view the model block-wise, the operators $\Phi$ and $\Psi$ share the properties stated above. Hence, based on the operator formulation, we deduce that the nonlocal operator $\Theta$ preserves such symmetry properties as well.

To obtain the sum of $\theta$, we take the Fourier transform of Eq. $(2.14): \hat{\theta}(\xi) \hat{u}(\xi)=\hat{\bar{f}}(\xi)$. The idea is to find $u \in \ell^{2}(\mathbb{L})$ and $f \in \ell^{2}(\mathbb{L})$ such that $\hat{u}(0) \neq 0$ while $\hat{f}(0)=0$. For this purpose, consider a particular function $u \in \ell^{2}(\mathbb{L})$ as follows:

$$
u(x)= \begin{cases}1, & |x| \leq N H, \\ 0, & \text { otherwise }\end{cases}
$$

for a fixed $N>0$, and then with direct calculations, we get the corresponding force function $f$,

$$
f(x)= \begin{cases}0, & |x| \leq N H-2 \varepsilon \text { or }|x| \geq N H+3 \varepsilon, \\ K_{2} \varepsilon^{-2}, & |x|=N H-\varepsilon \\ \left(K_{1}+K_{2}\right) \varepsilon^{-2}, & |x|=N H \\ -\left(K_{1}+K_{2}\right) \varepsilon^{-2}, & |x|=N H+\varepsilon \\ -K_{2} \varepsilon^{-2}, & |x|=N H+2 \varepsilon .\end{cases}
$$

The rest is to compute $\hat{f}(0)$. To that end, we use the Fourier transform of Eq. (3.8). Notice here $u \in \ell^{2}\left(\mathbb{L}_{C G}\right)$, so we have the following:

$$
\begin{aligned}
& \hat{\theta}(0)=\sum_{y \in \mathbb{L}_{C G}} \theta(y), \\
& \hat{u}(0)=\sum_{|y| \leq N H} 1=2 N+1, \\
& \hat{f}(0)=\varepsilon^{2}\left(K_{1}+K_{2},-\left(K_{1}+K_{2}\right),-K_{2}, 0, \cdots, 0, K_{2}\right)_{M \times 1}^{*} .
\end{aligned}
$$




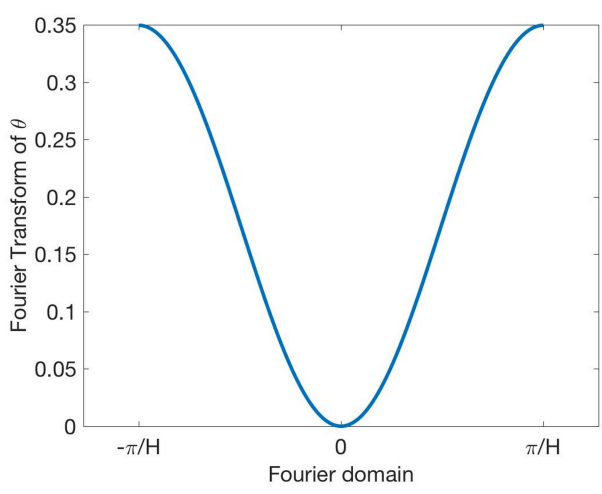

(a) $\hat{\theta}(\xi): K_{1}=1.0, K_{2}=0.2$

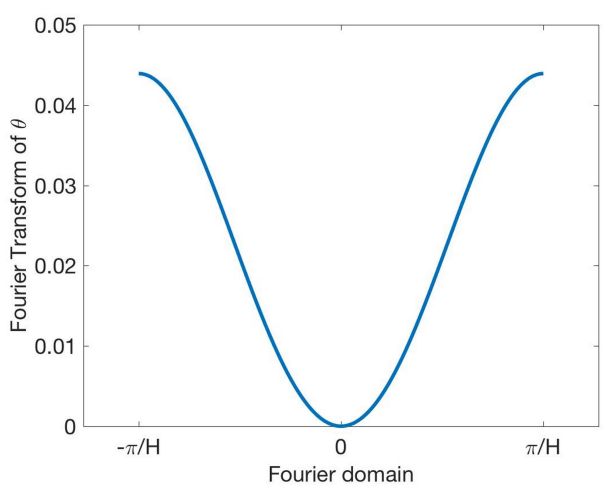

(b) $\hat{\theta}(\xi): K_{1}=1.0, K_{2}=-0.2$

Figure 2: Fourier transform of the nonlocal kernel function $\theta$. Since $\theta$ is defined for atoms on the coarse lattice, its Fourier transform is periodic with a period $2 \pi / H$. Here $M=20, \varepsilon=1$.

A direct calculation of matrices gives

$$
\left(\phi \mathcal{F}(L)(0) \psi^{*}\right)\left(\psi \mathcal{F}(L)(0) \psi^{*}\right)^{-1}=(-1, \cdots,-1)_{1 \times(M-1)} .
$$

So we obtain the Fourier transform of right side of Eq. (3.8),

$$
\mathcal{F}\left(\Phi f-\left(\Phi \circ L \circ \Psi^{*}\right)\left(\Psi \circ L \circ \Psi^{*}\right)^{-1} \circ \Psi f\right)=(1, \cdots, 1) \hat{f}(0)=0 .
$$

Therefore, $\hat{u}(0)$ is nonzero and $\hat{f}(0)=0$. Therefore, $\hat{\theta}(0)=0$.

\subsection{Spatial decay of the nonlocal kernel}

Our next result provides some insight into the spatial decay of the nonlocal kernel function $\theta$ defined in Theorem 4.1.

Theorem 4.2. For any integer $s \in \mathbb{N}$, as $y \rightarrow \infty, \theta$ satisfies

$$
\theta(y)=o\left(|y|^{-s}\right) \text {. }
$$

Proof. We shall look at the decay rate via the Fourier transform $\hat{\theta}$. By Lemma 3.2, $\psi \mathcal{F}(L) \psi^{*} \in\left(C^{\infty}(\mathbb{T})\right)^{(M-1) \times(M-1)}$ and is invertible. Therefore, the Fourier transform of Eq. (3.8) implies that $\hat{\theta} \in C^{\infty}(\mathbb{T})$.

Now we express the kernel function via the inverse Fourier transform of $\hat{\theta}$, and perform integration by parts to obtain

$$
\begin{aligned}
\theta(y) & =\frac{H}{2 \pi} \int_{-\frac{\pi}{H}}^{\frac{\pi}{H}} \hat{\theta}(\xi) e^{i y \xi} d \xi \\
& =(i y)^{-1} \frac{H}{2 \pi}\left[\hat{\theta}\left(\frac{\pi}{H}\right) e^{i y \frac{\pi}{H}}-\hat{\theta}\left(\frac{\pi}{H}\right) e^{i y \frac{\pi}{H}}\right]-(i y)^{-1} \frac{H}{2 \pi} \int_{-\frac{\pi}{H}}^{\frac{\pi}{H}} e^{i y \xi} d \hat{\theta}(\xi)
\end{aligned}
$$




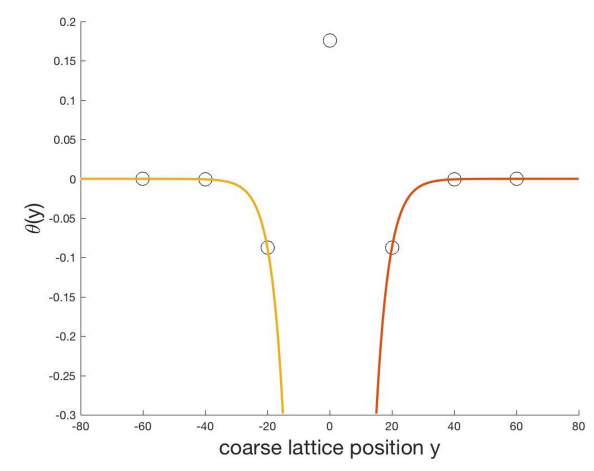

(a) $\theta, K_{1}=1, K_{2}=0.2$

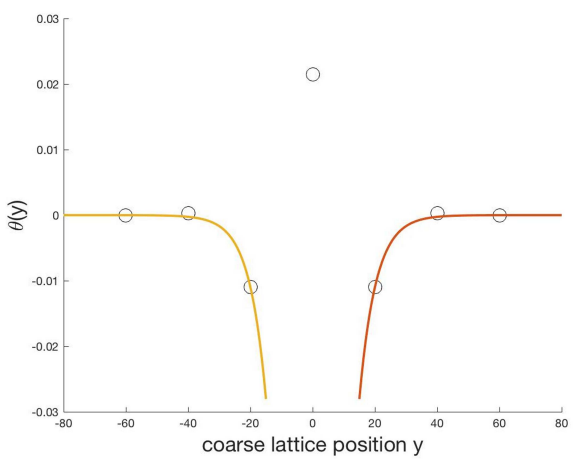

(c) $\theta, K_{1}=1, K_{2}=-0.2$

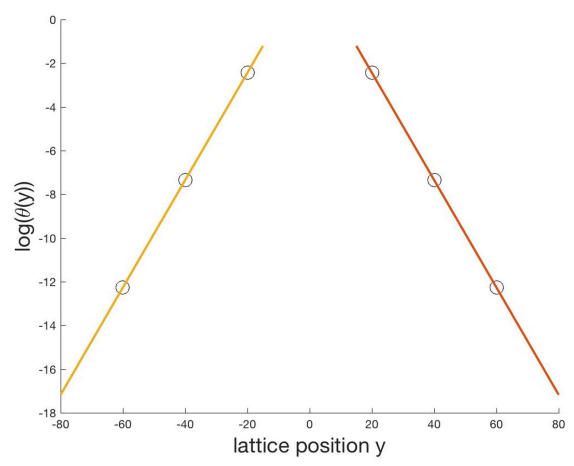

(b) $\log (|\theta|), K_{1}=1, K_{2}=0.2$

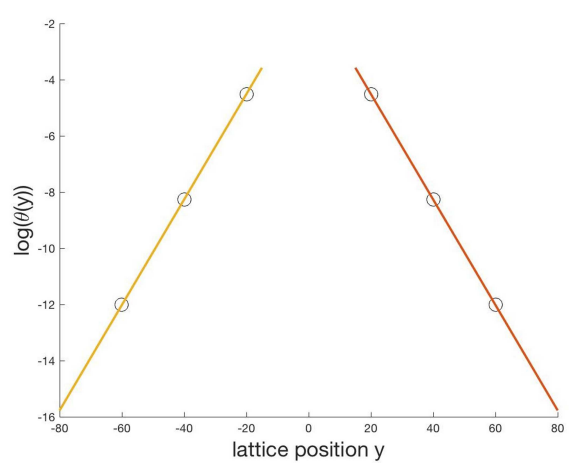

(d) $\log (|\theta|), K_{1}=1, K_{2}=-0.2$

Figure 3: The figures illustrate $\theta$ and the corresponding exponential fitting except at $y=0$ (which plays no role in the nonlocal form). Here $M=20, \varepsilon=1$. Notice that $\theta$ may take positive values if $K_{2}<0$, e.g., at $y=-40,40$ in the third figure.

$$
=-(i y)^{-1} \frac{H}{2 \pi} \int_{-\frac{\pi}{H}}^{\frac{\pi}{H}} e^{i y \xi} \hat{\theta}^{\prime}(\xi) d \xi
$$

This implies that $\theta(y)=\mathcal{O}\left(|y|^{-1}\right)$ since the integrand is bounded in $y$. We can proceed with arbitrary order since $\hat{\theta}$ is smooth and periodic. This completes the proof.

Remark 4.1. Our numerical test suggests that the nonlocal kernel function $\theta$ decays exponentially. See Fig. 3. The same behavior has been observed in the context of dynamic coarse-graining [3].

\subsection{Monotonicity and maximum principle with repulsive interactions}

Next we restrict our attention to the case where $K_{2}>0$. The operator $L: \ell^{2}(\mathbb{L}) \rightarrow \ell^{2}(\mathbb{L})$ can be viewed as a discretization of an elliptic operator, for which the maximum principle 
holds. We shall show similar properties for the reduced equation (2.14) in the following theorems. For additional discussions on monotone properties and maximum principles in the context of nonlocal models, we refer to [31,32].

Theorem 4.3. Given $f \in \operatorname{Image}(L)$ and $f \geq 0$, there is a unique $u \in \ell^{2}(\mathbb{L})$ such that $u(x) \geq 0$ for all $x \in \mathbb{L}$ and $u$ solves Eq. (2.11). As a consequence, if $\bar{f} \in \operatorname{Image}(\Theta)$ and $\bar{f} \geq 0$, then there is a unique $v \in \ell^{2}\left(\mathbb{L}_{C G}\right)$ such that $v(y) \geq 0$ for all $y \in \mathbb{L}_{C G}$, and $v$ solves $E q$. (2.14).

Proof. $L$ is a linear operator, associated with the equation,

$$
-K_{1} \frac{u(x+\varepsilon)-2 u(x)+u(x-\varepsilon)}{\varepsilon^{2}}-K_{2} \frac{u(x+2 \varepsilon)-2 u(x)+u(x-2 \varepsilon)}{\varepsilon^{2}}=0 .
$$

The characteristic equation of the above is of fourth order with roots,

$$
\lambda_{1}, \lambda_{2}=1, \quad \lambda_{3}, \lambda_{4}=\frac{\left(-K_{1}-2 K_{2}\right) \pm \sqrt{K_{1}^{2}+4 K_{1} K_{2}}}{2 K_{2}} .
$$

When $K_{2}>0$, all roots are real. Thus the solution $u \in \operatorname{Kernel}(L)$ is a combination of an affine function and powers of $\lambda_{3}, \lambda_{4}$. However, the fact that $u \in \ell^{2}(\mathbb{L})$ implies that $u \equiv 0$. So for any $f \in \operatorname{Image}(L)$, the solution $u$ is unique.

We now prove that $\inf \{u(x): x \in \mathbb{L}\} \geq 0$ under the condition that $\bar{f} \geq 0$. Suppose on the contrary there exists $u\left(x_{0}\right)<0$, we use the equation at $x=x_{0}$,

$$
\begin{aligned}
& -K_{1}\left(u\left(x_{0}+\varepsilon\right)-2 u\left(x_{0}\right)+u\left(x_{0}-\varepsilon\right)\right) \\
& -K_{2}\left(u\left(x_{0}+2 \varepsilon\right)-2 u\left(x_{0}\right)+u\left(x_{0}-2 \varepsilon\right)\right) \\
= & K_{1}\left(u\left(x_{0}\right)-u\left(x_{0}+\varepsilon\right)\right)+K_{1}\left(u\left(x_{0}\right)-u\left(x_{0}-\varepsilon\right)\right) \\
& K_{2}\left(u\left(x_{0}\right)-u\left(x_{0}+2 \varepsilon\right)\right)+K_{2}\left(u\left(x_{0}\right)-u\left(x_{0}-2 \varepsilon\right)\right) \\
= & f\left(x_{0}\right) \geq 0 .
\end{aligned}
$$

Since $K_{1}, K_{2}>0$, either all of the four differences are 0 , which means $u(x)=u\left(x_{0}\right)$ for all $x$, or one of the differences is positive, meaning there exists some $x_{1} \neq x_{0}$ such that $u\left(x_{1}\right)<u\left(x_{0}\right)<0$. The former case is not possible since $u \in \ell^{2}(\mathbb{L})$. In the latter case, we can repeat the argument for $u\left(x_{1}\right)$ and obtain a sequence of negative values all less than $u\left(x_{0}\right)$, which is a contradiction too.

Now for $\bar{f} \in \ell^{2}\left(\mathbb{L}_{C G}\right)$ and $\bar{f} \geq 0$, we simply take $f=\Phi^{*} \bar{f}$, so $f \geq 0$, which implies $u \geq 0$ and therefore $v=\Phi u \geq 0$.

Theorem 4.3 is equivalent to the strong maximum principle stating that any solution to Eq. (2.14) cannot take maximum value except the 0 function.

Theorem 4.4. Suppose $K_{1}, K_{2}>0$, then the nonlocal kernel function $\theta(y) \leq 0$ for any $y \in$ $\mathbb{L}_{C G} \backslash\{0\}$. 
Proof. Let $\left\{e_{i}: i \in \mathbb{Z}\right\}$ be the standard basis of $\ell^{2}(\mathbb{L})$. Since $\Theta$ is a Toeplitz operator generated by $\theta$, according to Eq. (3.5), we only need to show that for $i \neq j$,

$$
\left(e_{i}, \Theta e_{j}\right)_{\ell^{2}\left(\mathbb{L}_{C G}\right)}=\left(e_{i},\left(\Phi \circ L \circ \Phi^{*}\right)-\left(\Phi \circ L \circ \Psi^{*}\right)\left(\Psi \circ L \circ \Psi^{*}\right)^{-1}\left(\Psi \circ L \circ \Phi^{*}\right) e_{j}\right)_{\ell^{2}\left(\mathbb{L}_{C G}\right)} \leq 0 .
$$

By definition, $\left(e_{i}, \Phi \circ L \circ \Phi^{*} e_{j}\right)=0$. Meanwhile, $\left(\Phi \circ L \circ \Psi^{*}\right)$ is a block-wise Toeplitz operator generated by

$$
\left(\cdots, 0, X_{1}, X_{2}, 0 \cdots\right)
$$

where

$$
\begin{aligned}
& X_{1}=\left(0, \cdots, 0,-K_{2},-K_{1}\right)_{1 \times(M-1)}, \\
& X_{2}=\left(-K_{1},-K_{2}, 0, \cdots, 0\right)_{1 \times(M-1)} .
\end{aligned}
$$

Therefore, we have $\left(\Psi \circ L \circ \Phi^{*}\right) e_{i} \leq 0$. Using the same argument as in the proof of Theorem 4.3 , we can show that if $w \in \ell^{2}\left(\mathbb{L}_{C G}\right)$ satisfies

$$
\left(\Psi \circ L \circ \Psi^{*}\right) w=\left(\Psi \circ L \circ \Phi^{*}\right) e_{j}
$$

then $w(y) \geq 0$ for $\forall y \in \mathbb{L}_{C G} \backslash\{0\}$. Since $\Psi \circ L \circ \Psi^{*}$ is invertible, eventually we get

$$
\left(\left(\Psi \circ L \circ \Phi^{*}\right) e_{i},\left(\Psi \circ L \circ \Psi^{*}\right)^{-1}\left(\Psi \circ L \circ \Phi^{*}\right) e_{j}\right)_{\ell^{2}\left(\mathbb{L}_{C G}\right)} \geq 0 .
$$

Combining all the facts, the theorem is proved.

\subsection{Rescaling property}

Finally we observe the rescaling property of the nonlocal kernel function $\theta$, an important assumption in nonlocal modeling such as in peridynamics, see [26,27].

To establish the rescaling equation, we need a pivoting constant. Notice that Eq. (2.11) corresponds to the Poisson equation (2.12) with elastic constant $K_{1}+4 K_{2}$. The reduced equation (2.14) is expected to have the same associated elastic constant.

Conceptually, if one takes $u(x)=-x^{2} / 2$, then $L u=f=\left(\cdots, K_{1}+4 K_{2}, K_{1}+4 K_{2}, \cdots\right) \in$ $\ell^{\infty}(\mathbb{L})$. Although the coarse-graining is done in the $\ell^{2}$ space, we assume that it is true for such $u(x)$ and $f(x)$ and the Fourier transforms are interpreted in the sense of distributions. Eq. (4.8) shows that the coarse-graining process on the force function $f$ is in fact summing up $f$ on $\mathbb{L}$ for every $M$ atoms to get $\bar{f}$. Therefore, we postulate the following identity relating to the elastic constant:

$$
-\frac{1}{2 M} \sum \theta(y) y^{2}=K_{1}+4 K_{2},
$$

which has been verified numerically. 


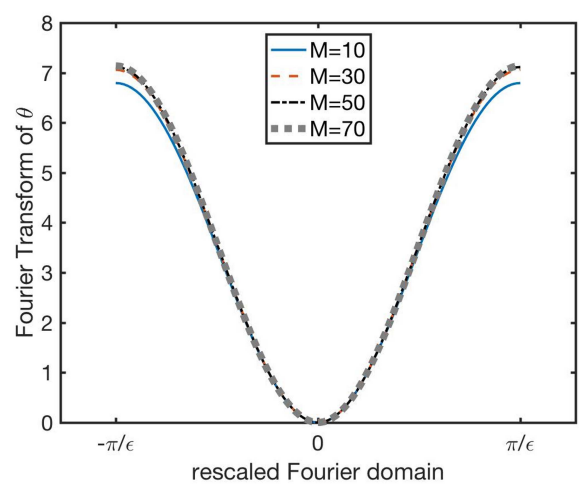

(a) $K_{1}=1.0, K_{2}=0.2$

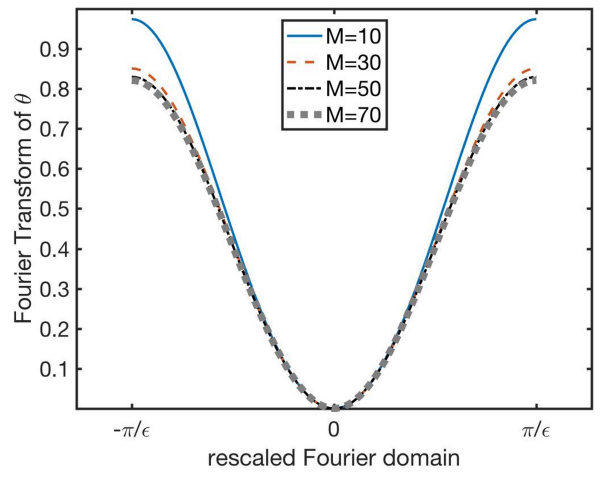

(b) $K_{1}=1.0, K_{2}=-0.2$

Figure 4: $M \hat{\theta}(\xi / M)$ for $M=10,30,50,70$. The rescaling property asserts that as $M \rightarrow \infty, M \hat{\theta}(\xi / M)$ approaches a limit function on $(-\pi / \varepsilon, \pi / \varepsilon)$. Here $\varepsilon=1$.

Now assuming (4.17) is true for all $M$, and there exists a function $\theta_{0}$ such that

$$
-\frac{1}{2} \sum_{x \in \mathbb{L}} \theta_{0}(x) x^{2}=-\frac{1}{2 M} \sum_{y \in \mathbb{L}_{C G}} \theta(y) y^{2}=-\frac{1}{2} \sum_{x \in \mathbb{L}} M \theta(M x) x^{2}
$$

holds for all sufficiently large $M$, we let $\theta_{0}(x)=M \theta(M x)$ for $x \in \mathbb{L}$.

Under this assumption, the Fourier transform gives a similar rescaling,

$$
\hat{\theta}_{0}(\xi)=\sum_{x \in \mathbb{L}} M \theta(M x) e^{-i x \xi}=M \sum_{y \in \mathbb{L}_{C G}} \theta(y) e^{-i y \xi / M}=M \hat{\theta}\left(\frac{\xi}{M}\right),
$$

for $\xi \in(-\pi / \varepsilon, \pi / \varepsilon)$.

As is shown in Fig. 4, when $M$ increases, the rescaled function $M \hat{\theta}(\xi / M)$ indeed converges to a fixed function, substantiating the assumption on the existence of $\theta_{0}$. Thus, $M$ serves as a parameter for the characteristic range of nonlocal interactions. Following the literature on nonlocal mechanics, particularly peridynamics [26,27], we may view $M$ as the effective nonlocal horizon parameter. Naturally, the particular form of rescaled nonlocal interaction kernel $\theta_{0}$ and the effective horizon parameter $M$ are not only determined by $M$ but also the original model and the restriction operators $\Phi$ and $\Psi$.

\section{Connection to continuum models}

Due to the non-locality of the kernel function $\theta$, it is natural to think of its consistency with a continuum model which is also a nonlocal model, i.e., a nonlocal PDE. This leads us to attempt to make a connection to the peridynamic models, recently developed to model nonlocal material interactions $[28,29]$. 
In order to obtain desired limiting behavior as the horizon parameter tends to 0 , it has been widely accepted that the nonlocal kernel function, which indicates the relative strength of interactions within the horizon, is chosen to be a positive non-increasing function that approaches a Dirac delta function in the sense of distribution as the horizon tends to zero, see for example, [1]. Moreover, it has been observed in [22] that to be compatible with the local models and obtain desired compactness, the kernel function must meet certain growth conditions. However, even with these constraints, there are still many choices of such kernel functions. Widely used nonlocal kernel functions in numerical simulations include Gaussian kernels [5], constant kernel [9], power law kernel $[10,11,32]$, or others, [1]. On the other hand, it is of practical importance to identify kernels based on the underlying material structures and atomic interactions.

Let us start with the reduced equation in the Fourier space,

$$
\hat{\theta}(\xi) \hat{u}(\xi)=\hat{f}(\xi), \xi \in\left(-\frac{\pi}{H}, \frac{\pi}{H}\right) .
$$

$\hat{\theta}(\xi)$ is a periodic function. For the continuum approximation, we aim to approximate $\hat{\theta}(\xi)$ for small wave number $\xi$ (long wave length). In particular, we choose the Padé approximation since it has the well-known stability property and larger convergence radius when compared to Taylor expansions. In addition, to enhance the stability of the approximation, we would like to control the bound of $\hat{\theta}(\xi)$ for large $\xi$. Rational approximations are clearly good candidates for this purpose.

Therefore, we propose to choose the following type of rational functions:

$$
R[m / n](\xi)=\frac{a_{0}+a_{1} \xi+a_{2} \xi^{2}+\cdots+a_{m} \xi^{m}}{b_{0}+b_{1} \xi+b_{2} \xi^{2}+\cdots+b_{n} \xi^{n}} .
$$

When the kernel function is approximated by rational functions, and also when $\xi$ is extended to the entire $\mathbb{R}$, the reduced model will correspond to local PDEs after an inverse Fourier transform over $\mathbb{R}$. For example, if $n=m=2$ and we choose

$$
R[2 / 2](\xi)=\frac{a_{0}+a_{2} \xi^{2}}{b_{0}+b_{2} \xi^{2}}
$$

Such an approximation would replace Eq. (5.1) by

$$
\left(a_{0}+a_{2} \xi^{2}\right) \hat{u}(\xi)=\left(b_{0}+b_{2} \xi^{2}\right) \hat{f}(\xi), \quad \forall \xi \in \mathbb{R},
$$

which corresponds the PDE

$$
a_{0} U(x)-a_{2} U^{\prime \prime}(x)=b_{0} \bar{F}(x)-b_{2} \bar{F}^{\prime \prime}(x), \quad \forall x \in \mathbb{R} .
$$

There are a couple of ways to specify the approximation conditions. However, in general, they may not lead to stable PDEs. For example, if one tries only to match $\hat{\theta}(\xi)$ at $\xi=0$ to arbitrary order, the resulting PDEs might be ill-posed. 
These examples suggest that we ought to impose other types of constraints. We notice that $\hat{\theta}$ is an even function, so no odd order terms appear in rational approximations. Secondly, $\hat{\theta}$ is nonnegative, so we want approximations to stay nonnegative which is related to certain stability properties. Finally, $\hat{\theta}$ is periodic, so approximations should not blow up for large $\xi$.

Let us consider the following rational functions,

$$
\begin{aligned}
& R[2 / 2](\xi)=\frac{a_{0}+a_{2} \xi^{2}}{1+b_{2} \xi^{2}} \\
& R[2 / 4](\xi)=\frac{a_{0}+a_{2} \xi^{2}}{1+b_{2} \xi^{2}+b_{4} \xi^{4}} \\
& R[4 / 4](\xi)=\frac{a_{0}+a_{2} \xi^{2}+a_{4} \xi^{4}}{1+b_{2} \xi^{2}+b_{4} \xi^{4}}
\end{aligned}
$$

To obtain the coefficients in the rational functions that eventually lead to stable PDE models, we impose the following conditions.

$$
\begin{aligned}
& R(0)=\hat{\theta}(0), \\
& R^{\prime}(0)=\hat{\theta}^{\prime}(0), \\
& R^{\prime \prime}(0)=\hat{\theta}^{\prime \prime}(0), \\
& R\left(\frac{\pi}{H}\right)=\hat{\theta}\left(\frac{\pi}{H}\right), \\
& R^{\prime}\left(\frac{\pi}{H}\right)=\hat{\theta}^{\prime}\left(\frac{\pi}{H}\right), \\
& \lim _{\xi \rightarrow \infty} R(\xi)=\frac{H}{2 \pi} \int_{-\frac{\pi}{H}}^{\frac{\pi}{H}} \hat{\theta}(\xi) d \xi .
\end{aligned}
$$

We now explain the purpose of these constraints. First, the conditions (5.9a) and (5.9b) require that the rational function matches the behavior of $\hat{\theta}$ around $\xi=0$. Secondly, since the second order derivative of $\hat{\theta}$ at $\xi=0$ is related to the elastic constant of the model, we enforce (5.9c). Thirdly, we enforce the approximation functions to match the values at the boundary of the Fourier domain $(-\pi / H$ or $\pi / H)$, i.e., (5.9d) and (5.9e). Finally, if more parameters are permitted, we can also control the behavior of $R[\mathrm{~m} / \mathrm{n}]$ for high frequencies. For instance, we impose (5.9e), or use the average of $\hat{\theta}$ function in one period, as in (5.9f).

For the specific approximation $R[2 / 2]$ in (5.6), it is determined from the conditions (5.9a), (5.9b), and (5.9c); the approximation $R[2 / 4](5.7)$ is determined based on (5.9a), $(5.9 \mathrm{~b}),(5.9 \mathrm{c})$ and $(5.9 \mathrm{~d})$; The approximation $R[4 / 4]$ (5.8) satisfies all the conditions.

With the above-mentioned conditions, it can be shown that the resulting partial differential equations have guaranteed stability. We will only include the proof for the stability of the PDE associated with $R[4 / 4]$ in (5.8). 


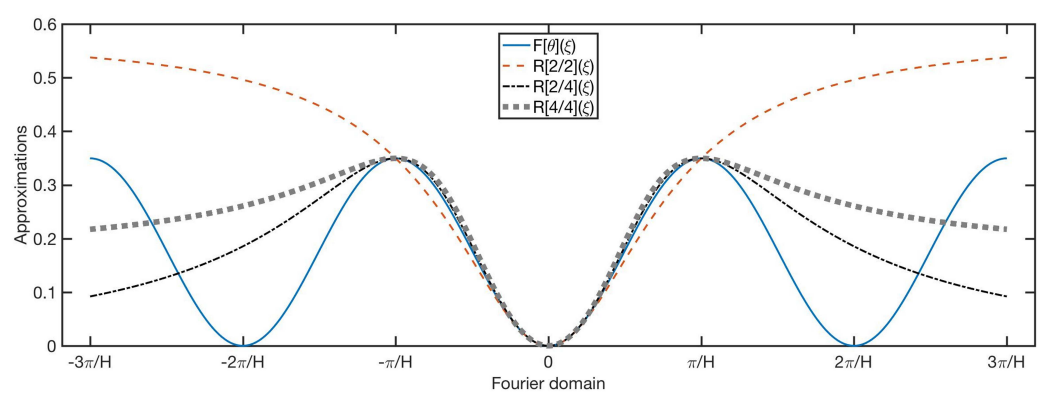

(a) $K_{1}=1, K_{2}=0.2$

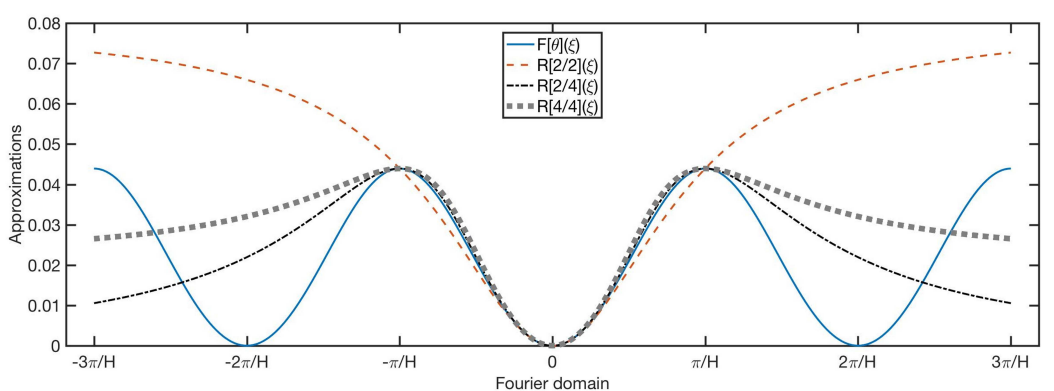

(b) $K_{1}=1, K_{2}=-0.2$

Figure 5: Approximations of $\hat{\theta}(\xi)$ : we are mostly concerned about the approximation errors within one period, while guaranteeing boundedness toward infinity. Meanwhile, the positivity of $\hat{\theta}(\xi)$ is maintained by assigning conditions at $-\pi / H$ and $\pi / H$, as is proven in Theorem 5.1. For this numerical test, we chose $M=20, \varepsilon=1$.

Theorem 5.1. The PDE model from the rational approximation $R[4 / 4]$ is given by

$$
a_{0} U(x)-a_{2} U^{\prime \prime}(x)+a_{4} U^{(4)}(x)=\bar{F}(x)-b_{2} \bar{F}^{\prime \prime}(x)+b_{4} \bar{F}^{(4)}(x) .
$$

If $R[4 / 4]$ satisfies all the conditions listed in (5.9), then $a_{0}=0, a_{2}, a_{4}>0, b_{4}>0$. Consequently, the following energy estimate holds:

$$
\int_{\mathbb{R}} a_{2}\left|U^{\prime}\right|^{2}+a_{4}\left|U^{\prime \prime}\right|^{2} d x \leq\left(\int_{\mathbb{R}}|\bar{F}|^{2}+\left|b_{2}\right|\left|\bar{F}^{\prime \prime}\right|^{2}+b_{4}\left|\bar{F}^{4}\right|^{2} d x\right)^{\frac{1}{2}} \cdot\left(\int_{\mathbb{R}}|U|^{2} d x\right)^{\frac{1}{2}} .
$$

Proof. Eq. (5.10) is a direct consequence of the inverse Fourier transform over $\mathbb{R}$.

Regarding the stability, we show the energy estimate. From Theorem 4.1, we have $\hat{\theta}(0)=0$, so condition (5.9a) implies that $R[4 / 4](0)=a_{0}=0$. Next we consider (5.9c). From the Fourier transform, we find that,

$$
\hat{\theta}^{\prime \prime}(0)=-\sum_{y \in \mathbb{L}_{C G}} \theta(y) y^{2} .
$$

Theorem 4.4 implied that $\theta(y) \leq 0$ for all $y \neq 0$. Therefore, $R[4 / 4]^{\prime \prime}(0)=a_{2}>0$. 
Suppose now $a_{4}<0, a_{2} \xi^{2}+a_{4} \xi^{4}$ has roots when $\xi>0$. However, as $\xi \rightarrow \infty, R[4 / 4](\xi) \rightarrow$ $a_{4} / b_{4}>0$ due to (5.8) and (5.9f). That means $a_{2} \xi^{2}+a_{4} \xi^{4}$ must have more than two roots when $\xi>0$, or one root at $\xi_{0}=\sqrt{-a_{2} / a_{4}}$ satisfying $R[4 / 4]^{\prime}\left(\xi_{0}\right)=0$. The former case can not be consistent with $a_{2} \xi^{2}+a_{4} \xi^{4}$. In the latter case, for $R[4 / 4](\xi)$ to be nonnegative around $\xi_{0}, 1+b_{2} \xi^{2}+b_{4} \xi^{4}$ must contain the factor $1+\left(a_{4} / a_{2}\right) \xi^{2}$, or otherwise it is easy to find that $R[4 / 4]^{\prime}\left(\xi_{0}\right) \neq 0$. Thus $R[4 / 4](\xi)$ is degenerate since

$$
R[4 / 4](\xi)=\frac{a_{2} \xi^{2}+a_{4} \xi^{4}}{1+b_{2} \xi^{2}+b_{4} \xi^{4}}=\frac{a_{2} \xi^{2}\left(1+a_{4} / a_{2} \xi^{2}\right)}{\left(1+b^{\prime} \xi^{2}\right)\left(1+a_{4} / a_{2} \xi^{2}\right)}
$$

which is impossible to meet all conditions in (5.9) due to the lack of degrees of freedom. Therefore, $a_{4}>0$ and $b_{4}>0$.

Now we multiply both sides of Eq. (5.10) by $U(x)$, integrate by parts and then apply Cauchy's inequality. We obtain

$$
\begin{aligned}
& \int_{\mathbb{R}} a_{2}\left|U^{\prime}\right|^{2} d x+\int_{\mathbb{R}} a_{4}\left|U^{\prime \prime}\right|^{2} d x \\
= & -\int_{\mathbb{R}}\left(\bar{F}-b_{2} \bar{F}^{\prime \prime}+b_{2} \bar{F}^{(4)}\right) U d x \\
\leq & \left(\int_{\mathbb{R}}|\bar{F}|^{2}+\left|b_{2}\right|\left|\bar{F}^{\prime \prime}\right|^{2}+b_{4}\left|\bar{F}^{4}\right|^{2} d x\right)^{\frac{1}{2}} \cdot\left(\int_{\mathbb{R}}|U|^{2} d x\right)^{\frac{1}{2}} .
\end{aligned}
$$

The proof is completed.

In general the kernel functions for the nonlocal continuous PDE model can be found explicitly. In the case when $1+b_{2} \xi^{2}+b_{4} \xi^{4}$ can be factorized, we have $b_{2} \geq 0$ since $1+$ $b_{2} \xi^{2}+b_{4} \xi^{4}$ must not have real zeros by construction. Now by using partial fractions, the rational functions can be written as

$$
\begin{aligned}
& R[2 / 2]=\frac{s_{1}}{1+t_{1} \xi^{2}}+s_{2}, \\
& R[2 / 4]=\frac{s_{1}}{1+t_{1} \xi^{2}}+\frac{s_{2}}{1+t_{2} \xi^{2}}, \\
& R[4 / 4]=\frac{s_{1}}{1+t_{1} \xi^{2}}+\frac{s_{2}}{1+t_{2} \xi^{2}}+s_{3},
\end{aligned}
$$

where $s_{1}, s_{2}$, and $s_{3}$ are coefficients obtained from the partial fraction procedure, and $t_{1}, t_{2}>0$ since $b_{2}, b_{4} \geq 0$.

Now, applying the inverse Fourier transform to the functions in (5.14), we find

$$
\begin{aligned}
& P[2 / 2](x)=\frac{s_{1}}{2 \sqrt{t_{1}}} e^{-|x| / \sqrt{t_{1}}}+s_{2} \delta(x), \\
& P[2 / 4](x)=\frac{s_{1}}{2 \sqrt{t_{1}}} e^{-|x| / \sqrt{t_{1}}}+\frac{s_{2}}{2 \sqrt{t_{2}}} e^{-|x| / \sqrt{t_{2}},} \\
& P[4 / 4](x)=\frac{s_{1}}{2 \sqrt{t_{1}}} e^{-|x| / \sqrt{t_{1}}}+\frac{s_{2}}{2 \sqrt{t_{2}}} e^{-|x| / \sqrt{t_{2}}}+s_{3} \delta(x) .
\end{aligned}
$$


Therefore, rational approximation is by nature an approximation of the kernel function via exponential function $e^{-\alpha|x|}$ or Dirac delta distribution.

\section{Conclusion}

One of our motivations for the present study is the recent interests in nonlocal mechanical models like peridynamics [25] and related discussions on the origins of nonlocality $[8,29]$. It has been suggested that two sources of non-locality may contribute to the long-range interactions in the peridynamic formulations, namely coarse-graining as in [28] and homogenization as in [29]. Discussions along this line, within the context of multiscale modeling, have been further explored in [8]. Indeed, delicate treatment is needed to gain more mathematical insight from the underlying atomistic models, since existing descriptions (e.g. [28,29]) have largely remained procedural.

In this paper, we posed a one-dimensional linear molecular model, and reduced it via coarse-graining while retaining all information from the full model. This is similar to the procedure in [28], although the approach in [28] was done for continuum models. Both the local and nonlocal interactions were considered, and detailed derivations using the lattice Green's functions and Schur complements were shown. The reduced model involves a discrete nonlocal kernel function $\theta$ that exhibits fast decay. Subsequently, we drew connections from discrete nonlocal kernel functions to the continuous ones. Through proper Padé approximations we were able to prove the stability of the corresponding continuum models.

Apparently there are a lot of possible extensions to our results for future works. When we approximate $\hat{\theta}$ in Section 5, many other interesting options are open in addition to rational functions. For example, the Gaussian kernel, which is very popular in nonlocal models due to its smoothness, fast decay, uniformity among other desired properties, can fit in the current framework. By approximating $\hat{\theta}$ with a Gaussian function (flipped at 0 ), one obtains the Fourier transform of a nonlocal continuum model with a Gaussian kernel. This may shed light upon and provide support to the modeling procedure for peridynamics where the connection between microscopic and macroscopic models are crucial. In this work, such connections are made by matching the Fourier transform, which can be done for several nonlocal kernels, see Fig. 6 for a schematic illustration.

Finally, the analysis in this work has been limited to the one-dimensional case. Naturally, extensions to particle interactions in higher dimensional settings and systems involving defects will be of much practical interests. For instance, the lattice Green's function has been used in two-dimensional systems $[17,20]$ to derive computationally more tractable reduced models. In addition, the analysis in [19] shows that the asymptotic behavior of the Green's function depends on the dimension as well as the lattice structure. The strong connection between the kernel and the Green's function suggests that the resulting nonlocal interactions will inherit similar dependence. The more general discussion will be dependent on the topological and geometrical structures of the underlying lattice and defect structures, thus leading to new challenges in the mathematical analysis. 


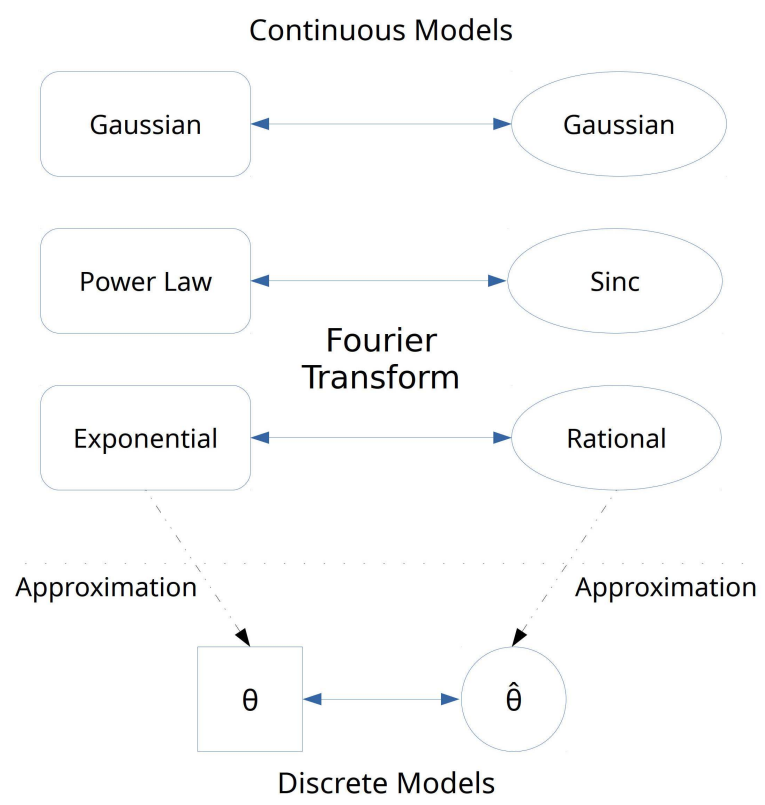

Figure 6: An illustration for approximating $\hat{\theta}$ by other types of functions that correspond to widely used nonlocal kernels.

\section{Acknowledgments}

The author XL would like to acknowledge the support from the NSF grant DMS-1522617. The work of QD is supported in part by NSF DMS-1719699 and Army Research Office (MURI W911NF-15-1-0562).

\section{Appendices}

\section{A Green's identities}

In the appendix, we establish the mathematical theory and provide rigorous treatment of the Green's identities toward coarse-graining. These formulas have been used to construct the atomistic-based boundary element methods [17,34].

The coarse-graining procedure relies on the discrete Green's identity. We first give,

Lemma A.1 (Summation by parts). Assume that $J_{A}$ and $J_{B}$ are integers, and $J_{B}-J_{A}>1$. There 
holds

$$
\begin{aligned}
& \sum_{j=J_{A}+1}^{J_{B}-1} \phi(j)(\psi(j+1)-\psi(j)) \\
= & \phi\left(J_{B}-1\right) \psi\left(J_{B}\right)-\phi\left(J_{A}\right) \psi\left(J_{A}+1\right)-\sum_{j=J_{A}+1}^{J_{B}-1}(\phi(j)-\phi(j-1)) \psi(j) .
\end{aligned}
$$

Similarly,

$$
\begin{aligned}
& \sum_{j=J_{A}+1}^{J_{B}-1} \phi(j)(\psi(j)-\psi(j-1)) \\
= & \phi\left(J_{B}\right) \psi\left(J_{B}-1\right)-\phi\left(J_{A}+1\right) \psi\left(J_{A}\right)-\sum_{j=J_{A}+1}^{J_{B}-1}(\phi(j+1)-\phi(j)) \psi(j) .
\end{aligned}
$$

For the difference terms involving next nearest neighbor, one has

$$
\begin{aligned}
& \sum_{j=J_{A}+1}^{J_{B}-1} \phi(j)(\psi(j+2)-\psi(j)) \\
= & \phi\left(J_{B}-1\right) \psi\left(J_{B}+1\right)+\phi\left(J_{B}-2\right) \psi\left(J_{B}\right)-\phi\left(J_{A}-1\right) \psi\left(J_{A}+1\right) \\
& -\phi\left(J_{A}\right) \psi\left(J_{A}+2\right) \sum_{j=J_{A}+1}^{J_{B}-1}(\phi(j)-\phi(j-2)) \psi(j),
\end{aligned}
$$

and similarly,

$$
\begin{aligned}
& \sum_{j=J_{A}+1}^{J_{B}-1} \phi(j)(\psi(j)-\psi(j-2)) \\
= & \phi\left(J_{B}+1\right) \psi\left(J_{B}-1\right)+\phi\left(J_{B}\right) \psi\left(J_{B}-2\right)-\phi\left(J_{A}+1\right) \psi\left(J_{A}-1\right) \\
& -\phi\left(J_{A}+2\right) \psi\left(J_{A}\right)-\sum_{j=J_{A}+1}^{J_{B}-1}(\phi(j+2)-\phi(j)) \psi(j) .
\end{aligned}
$$

Proof. For the summation by parts formulas, one only needs to make up boundaries and rearrange all terms.

Lemma A.2 (Green's identities). $J_{A}, J_{B}$ are integers and $J_{B}-J_{A}>1$. The following are Green's Identities for the difference operator involving next nearest neighbors:

$$
\begin{aligned}
& \sum_{j=J_{A}+1}^{J_{B}-1} \phi(j)(\psi(j+1)-2 \psi(j)+\psi(j-1)) \\
= & \sum_{j=J_{A}+1}^{J_{B}-1}(\phi(j+1)-2 \phi(j)+\phi(j-1)) \psi(j)+\phi\left(J_{A}+1\right) \psi\left(J_{A}\right) \\
& \quad+\phi\left(J_{B}-1\right) \psi\left(J_{B}\right)-\phi\left(J_{A}\right) \psi\left(J_{A}+1\right)-\phi\left(J_{B}\right) \psi\left(J_{B}-1\right),
\end{aligned}
$$


and

$$
\begin{aligned}
& \sum_{j=J_{A}+1}^{J_{B}-1} \phi(j)(\psi(j+2)-2 \psi(j)+\psi(j-2)) \\
& =\sum_{j=J_{A}+1}^{J_{B}-1}(\phi(j+2)-2 \phi(j)+\phi(j-2)) \psi(j)+\phi\left(J_{B}-1\right) \psi\left(J_{B}+1\right)+\phi\left(J_{A}+1\right) \psi\left(J_{A}-1\right) \\
& \quad+\phi\left(J_{B}-2\right) \psi\left(J_{B}\right)+\phi\left(J_{A}+2\right) \psi\left(J_{A}\right)-\phi\left(J_{B}+1\right) \psi\left(J_{B}-1\right) \\
& \quad-\phi\left(J_{A}-1\right) \psi\left(J_{A}+1\right)-\phi\left(J_{B}\right) \psi\left(J_{B}-2\right)-\phi\left(J_{A}\right) \psi\left(J_{A}+2\right) .
\end{aligned}
$$

Proof. Simply sum up the equations in Lemma A.1.

\section{B Derivations for Eq. (2.8) and Eq. (2.14)}

We show Eq. (2.8) holds. Using Green's identity, we compute

$$
\begin{aligned}
\varepsilon \sum_{x^{\prime} \in(y, y+H)} g\left(x, x^{\prime}\right) L u\left(x^{\prime}\right)= & \varepsilon \sum_{x^{\prime} \in(y, y+H)} \operatorname{Lg}\left(x, x^{\prime}\right) u\left(x^{\prime}\right) \\
& -\frac{K}{\varepsilon}[g(x, y) u(y+\varepsilon)+g(x, y+H) u(y+H-\varepsilon)] \\
& +\frac{K}{\varepsilon}[g(x, y+\varepsilon) u(y)+g(x, y+H-\varepsilon) u(y+H)] .
\end{aligned}
$$

Setting $x=y+\varepsilon$, we get

$$
\begin{aligned}
& u(y+\varepsilon)+\frac{K}{\varepsilon} g(y+\varepsilon, y) u(y+\varepsilon)+\frac{K}{\varepsilon} g(y+\varepsilon, y+H) u(y+H-\varepsilon) \\
= & \frac{K}{\varepsilon} g(y+\varepsilon, y+H-\varepsilon) u(y+H)+\varepsilon \sum_{x^{\prime} \in(y, y+H)} g\left(y+\varepsilon, x^{\prime}\right) f\left(x^{\prime}\right) .
\end{aligned}
$$

Similarly, we have

$$
\begin{aligned}
& u(y+H-\varepsilon)+\frac{K}{\varepsilon} g(y+H-\varepsilon, y) u(y+\varepsilon)+\frac{K}{\varepsilon} g(y+H-\varepsilon, y+H) u(y+H-\varepsilon) \\
= & \frac{K}{\varepsilon} g(y+H-\varepsilon, y+\varepsilon) u(y)+\varepsilon \sum_{x^{\prime} \in(y, y+H)} g\left(y+H-\varepsilon, x^{\prime}\right) f\left(x^{\prime}\right) .
\end{aligned}
$$


Now a direct substitution gives

$$
\begin{aligned}
& u(y+\varepsilon)=\left(1-\frac{\varepsilon}{H}\right) u(y)+\frac{\varepsilon}{H} u(y+H) \\
& -\frac{2 \varepsilon^{2}}{H-2 \varepsilon} \sum_{y \in(y, y+H)}\left[\frac{\varepsilon}{H} g(y+\varepsilon, y)+\left(1-\frac{\varepsilon}{H}\right) g(y+H-\varepsilon, y)\right] f(y) \\
& =\left(1-\frac{\varepsilon}{H}\right) u(y)+\frac{\varepsilon}{H} u(y+H)+\frac{\varepsilon^{2}}{K} \sum_{x \in(y, y+H)} \frac{H+y-x}{H} f(x), \\
& u(y+H-\varepsilon)=\frac{\varepsilon}{H} u(y)+\left(1-\frac{\varepsilon}{H}\right) u(y+H) \\
& -\frac{2 \varepsilon^{2}}{H-2 \varepsilon} \sum_{y \in(y, y+H)}\left[\left(1-\frac{\varepsilon}{H}\right) g(y+\varepsilon, y)+\frac{\varepsilon}{H} g(y+H-\varepsilon, y)\right] f(y) \\
& =\frac{\varepsilon}{H} u(y)+\left(1-\frac{\varepsilon}{H}\right) u(y+H)+\frac{\varepsilon^{2}}{K} \sum_{x \in(y, y+H)} \frac{x-y}{H} f(x) .
\end{aligned}
$$

By substituting $y$ to $y+H$, we find

$$
u(y-\varepsilon)=\frac{\varepsilon}{H} u(y-H)+\left(1-\frac{\varepsilon}{H}\right) u(y)+\frac{\varepsilon^{2}}{K} \sum_{x \in(y-H, y)} \frac{x-y+H}{H} f(x) .
$$

Notice that

$$
u(y+\varepsilon)-2 u(y)+u(y-\varepsilon)=-\frac{\varepsilon^{2}}{K} f(y) .
$$

Putting all equations together, there holds

$$
-K \frac{u(y+H)-2 u(y)+u(y-H)}{H^{2}}=\frac{\varepsilon}{H} \sum_{x \in(y-H, y+H)} h(x, y) f(x) .
$$

Coarse-grain model with nearest neighbor interactions is not of much interest. However, the Green's function idea can be easily extended to the case with next nearest neighbor interaction. Here the Green's function reads

$$
g\left(x-x^{\prime}\right)=\alpha\left|x-x^{\prime}\right|+\beta z^{\left|x-x^{\prime}\right| / \varepsilon}-\beta,
$$

where the parameters are given by

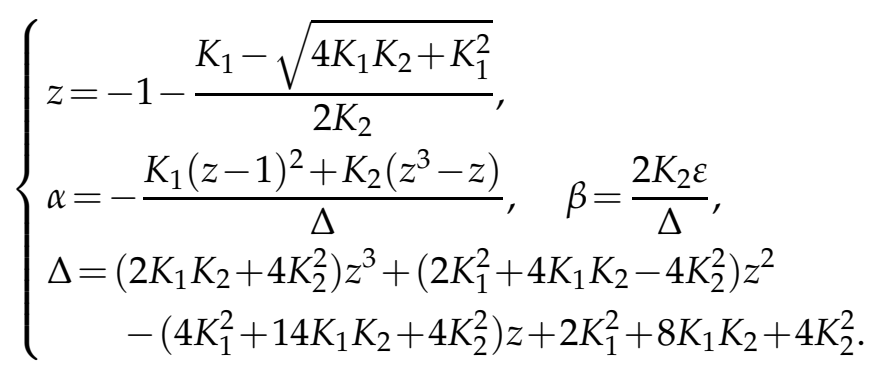


Then we get our hands on the model (2.11). It turns out that Green's identities eventually lead to a system of linear equations on $\mathbb{L}_{C G}$, which can be solved via Fourier transform.

Now simply compute

$$
\begin{aligned}
\varepsilon \sum_{x^{\prime} \in(y, y+H)} g\left(x, x^{\prime}\right) L u\left(x^{\prime}\right)= & \sum_{x^{\prime} \in(y, y+H)} L g\left(x, x^{\prime}\right) u\left(x^{\prime}\right) \\
& +K_{1} \varepsilon^{-1}[g(x, y) u(y+\varepsilon)+g(x, y+H) u(y+H-\varepsilon)] \\
& +K_{2} \varepsilon^{-1}[g(x, y-\varepsilon) u(y+\varepsilon)+g(x, y+H+\varepsilon) u(y+H-\varepsilon)] \\
& +K_{2} \varepsilon^{-1}[g(x, y) u(y+2 \varepsilon)+g(x, y+H) u(y+H-2 \varepsilon)] \\
& -K_{1} \varepsilon^{-1}[g(x, y+\varepsilon) u(y)+g(x, y+H-\varepsilon) u(y+H)] \\
& -K_{2} \varepsilon^{-1}[g(x, y+\varepsilon) u(y-\varepsilon)+g(x, y+H-\varepsilon) u(y+H+\varepsilon)] \\
& -K_{2} \varepsilon^{-1}[g(x, y+2 \varepsilon) u(y)+g(x, y+H-2 \varepsilon) u(y+H)] .
\end{aligned}
$$

By definition of Green's function, the following equation holds for all $x \in(y, y+H)$

$$
\begin{aligned}
u(x) & +\varepsilon^{-1}\left[K_{1} g(x, y)+K_{2} g(x, y-\varepsilon)\right] u(y+\varepsilon) \\
& +\varepsilon^{-1}\left[K_{1} g(x, y+H)+K_{2} g(x, y+H+\varepsilon)\right] u(y+H-\varepsilon) \\
& +\varepsilon^{-1} K_{2} g(x, y) u(y+2 \varepsilon)+\varepsilon^{-1} K_{2} g(x, y+H) u(y+H-2 \varepsilon) \\
& -\varepsilon^{-1} K_{2} g(x, y+\varepsilon) u(y-\varepsilon)-\varepsilon^{-1} K_{2} g(x, y+H-\varepsilon) u(y+H+\varepsilon) \\
=\varepsilon^{-1} & {\left[K_{1} g(x, y+\varepsilon)+K_{2} g(x, y+2 \varepsilon)\right] u(y) } \\
& +\varepsilon^{-1}\left[K_{1} g(x, y+H-\varepsilon)+K_{2} g(x, y+H-2 \varepsilon)\right] u(y+H) \\
& +\varepsilon \sum_{x^{\prime} \in(y, y+H)} g\left(x, x^{\prime}\right) f\left(x^{\prime}\right) .
\end{aligned}
$$

Notice that next nearest neighbor interactions bring in additional terms involving function values of atoms not on the coarse lattice.

For $y \in \mathbb{L}_{C G}$, we take $v(y)=u(y)$ and eliminate irrelevant terms $u(y+\varepsilon), u(y+2 \varepsilon)$, $u(y+H-\varepsilon)$ and $u(y+H-2 \varepsilon)$ to target $v(y)$. To that end we define a column vector $\mathbf{d}(y)=$ $(u(y+\varepsilon), u(y-\varepsilon), u(y+2 \varepsilon), u(y-2 \varepsilon))^{*}$.

After substitution, we obtain a system of equations

$$
A_{0} \mathbf{d}(y)+A_{1} \mathbf{d}(y+H)=\mathbf{b}_{0} v(y)+\mathbf{b}_{1} v(y+H)+\mathbf{f}_{0},
$$


where

$$
\begin{aligned}
& A_{0}=\left[\begin{array}{cccc}
1+\varepsilon^{-1}\left(K_{1} g(\varepsilon)+K_{2} g(2 \varepsilon)\right) & 0 & \varepsilon^{-1} K_{2} g(\varepsilon) & 0 \\
\varepsilon^{-1}\left(K_{1} g(2 \varepsilon)+K_{2} g(3 \varepsilon)\right) & -\varepsilon^{-1} K_{2} g(\varepsilon) & 1+\varepsilon^{-1} K_{2} g(2 \varepsilon) & 0 \\
\varepsilon^{-1}\left(K_{1} g(H-2 \varepsilon)+K_{2} g(H-\varepsilon)\right) & -\varepsilon^{-1} K_{2} g(H-3 \varepsilon) & \varepsilon^{-1} K_{2} g(H-2 \varepsilon) & 0 \\
\varepsilon^{-1}\left(K_{1} g(H-\varepsilon)+K_{2} g(H)\right) & -\varepsilon^{-1} K_{2} g(H-2 \varepsilon) & \varepsilon^{-1} K_{2} g(H-\varepsilon) & 0
\end{array}\right], \\
& A_{1}=\left[\begin{array}{cccc}
-\varepsilon^{-1} K_{2} g(H-2 \varepsilon) & \varepsilon^{-1}\left(K_{1} g(H-\varepsilon)+K_{2} g(H)\right) & 0 & \varepsilon^{-1} K_{2} g(H-\varepsilon) \\
-\varepsilon^{-1} K_{2} g(H-3 \varepsilon) & \varepsilon^{-1}\left(K_{1} g(H-2 \varepsilon)+K_{2} g(H-\varepsilon)\right. & 0 & \varepsilon^{-1} K_{2} g(H-2 \varepsilon) \\
-\varepsilon^{-1} K_{2} g(\varepsilon) & \varepsilon^{-1}\left(K_{1} g(2 \varepsilon)+K_{2} g(3 \varepsilon)\right) & 0 & 1+\varepsilon^{-1} K_{2} g(2 \varepsilon) \\
0 & 1+\varepsilon^{-1}\left(K_{1} g(\varepsilon)+K_{2} g(2 \varepsilon)\right) & 0 & \varepsilon^{-1} K_{2} g(\varepsilon)
\end{array}\right], \\
& \mathbf{b}_{0}=\left[\begin{array}{c}
\varepsilon^{-1} K_{2} g(\varepsilon) \\
\varepsilon^{-1} K_{1} g(\varepsilon) \\
\varepsilon^{-1}\left(K_{1} g(H-3 \varepsilon)+K_{2} g(H-4 \varepsilon)\right) \\
\varepsilon^{-1}\left(K_{1} g(H-2 \varepsilon)+K_{2} g(H-3 \varepsilon)\right)
\end{array}\right], \quad \mathbf{b}_{1}=\left[\begin{array}{c}
\varepsilon^{-1}\left(K_{1} g(H-2 \varepsilon)+K_{2} g(H-3 \varepsilon)\right) \\
\varepsilon^{-1}\left(K_{1} g(H-3 \varepsilon)+K_{2} g(H-4 \varepsilon)\right) \\
\varepsilon^{-1} K_{1} g(\varepsilon) \\
\varepsilon^{-1} K_{2} g(\varepsilon)
\end{array}\right], \\
& \mathbf{f}_{0}=\varepsilon \sum_{x^{\prime} \in(y, y+H)} f\left(x^{\prime}\right)\left(g\left(y+\varepsilon, x^{\prime}\right), g\left(y+2 \varepsilon, x^{\prime}\right), g\left(y+H-\varepsilon, x^{\prime}\right), g\left(y+H-2 \varepsilon, x^{\prime}\right)\right)^{T} .
\end{aligned}
$$

Formally the equation can be solved via Fourier transform,

$$
\begin{aligned}
& \hat{A}(\xi)=A_{0} e^{-i \xi H / 2}+A_{1} e^{i \xi H / 2}, \quad \hat{\mathbf{b}}(\xi)=\mathbf{b}_{0} e^{-i \xi H / 2}+\mathbf{b}_{1} e^{i \xi H / 2}, \\
& \hat{v}(\xi)=\sum_{y \in \mathbb{L}_{C G}} v(y) e^{-i y \xi}, \quad \hat{\mathbf{f}}_{0}(\xi)=\sum_{y \in \mathbb{L}_{C G}} \mathbf{f}_{0}(y) e^{-i y \xi},
\end{aligned}
$$

where $\xi \in(-\pi / H, \pi / H)$.

In Fourier space, the equation reads

$$
\hat{\mathbf{d}}(\xi)=\hat{A}(\xi)^{-1} \hat{\mathbf{b}}(\xi) \hat{v}(\xi)+\hat{A}(\xi)^{-1} \hat{\mathbf{f}}_{0}
$$

Since eventually we want to obtain an equation in $v(y)$ only, here we use the original equation to associate $\mathbf{d}$ with $v(y)$.

$$
\varepsilon^{-2} \mathbf{k} \cdot(\mathbf{1} v(y)-\mathbf{d}(y))=f(y),
$$

where $\mathbf{k}=\left(K_{1}, K_{1}, K_{2}, K_{2}\right)^{*}$.

Finally, we arrive at the equation,

$$
\mathcal{\varepsilon}^{-2}\left(2\left(K_{1}+K_{2}\right) \delta(y)-\mathbf{k} \mathcal{F}^{-1}\left(\hat{A}(\xi)^{-1} \hat{\mathbf{b}}(\xi)\right) v(y)\right)=f(y)+\mathcal{F}^{-1}\left(\hat{A}(\xi)^{-1} \hat{\mathbf{f}}_{0}\right),
$$

for $y \in \mathbb{L}_{C G}$. Here $\delta$ is the Kronecker Delta function, see (2.6). 


\section{References}

[1] R. Abeyaratne and O. Weckner. The effect of long-range forces on the dynamics of a bar. J. Mech. Phys. Solids, 53(3):705-728, 2005.

[2] A. Böttcher and S. M. Grudsky. Toeplitz Matrices, Asymptotic Linear Algebra, and Functional Analysis. Birkhäuser, 2012.

[3] W. Chu and X. Li. On the asymptotic behavior of the kernel function in the Generalized Langevin Equation: A one-dimensional lattice model. Journal of Statistical Physics, 170(2):378-398, 2018.

[4] P. G. Ciarlet. Discrete maximum principle for finite-difference operators. Aequationes mathematicae, 4(3):338-352, 1970.

[5] K. Dayal and K. Bhattacharya. Kinetics of phase transformations in the peridynamic formulation of continuum mechanics. Journal of the Mechanics and Physics of Solids, 54(9):1811-1842, 2006.

[6] M. Dobson and M. Luskin. An optimal order error analysis of the one-dimensional quasicontinuum approximation. SIAM Journal on Numerical Analysis, 47(4):2455-2475, 2009.

[7] Q. Du. Nonlocal Modeling, Analysis, and Computation, volume 94 of CBMS-NSF regional research conference series. SIAM, 2019.

[8] Q. Du, B. Engquist, and X. Tian. Multiscale modeling, homogenization and nonlocal effects: mathematical an d computational issues. Contemporary Mathematics, Celebrating 75 th Anniversary of Mathematics of Computation, 2020.

[9] Q. Du, Y. Tao, X. Tian, and J. Yang. Robust a posteriori stress analysis for quadrature collocation approximations of nonlocal models via nonlocal gradients. Computer Methods in Applied Mechanics and Engineering, 310:605-627, 2016.

[10] Q. Du and J. Yang. Fast and accurate implementation of fourier spectral approximations of nonlocal diffusion operators and its applications. Journal of Computational Physics, 332:118134, 2017.

[11] Q. Du, J. Yang, and Z. Zhou. Analysis of a nonlocal-in-time parabolic equation. Discrete $\mathcal{E}$ Continuous Dynamical Systems-Series B, 22(2), 2017.

[12] W. E and P. Ming. Cauchy-Born rule and the stability of crystalline solids: static problems. Archive for Rational Mechanics and Analysis, 183(2):241-297, 2007.

[13] S. Izvekov and G. A. Voth. Modeling real dynamics in the coarse-grained representation of condensed phase systems. J. Chem. Phys., 125:151101-151104, 2006.

[14] A. R. Leach. Molecular modelling: principles and applications. Prentice Hall, 2001.

[15] R. B. Lehoucq and S. A. Silling. Statistical coarse-graining of molecular dynamics into peridynamics. Report SAND2007-6410, Sandia National Laboratories, Albuquerque, New Mexico, 2007.

[16] X. Li. A coarse-grained molecular dynamics model for crystalline solids. International Journal for Numerical Methods in Engineering, 83(8-9):986-997, 2010.

[17] X. Li. An atomistic-based boundary element method for the reduction of the molecular statics models. Comp. Meth. Appl. Mech. Engreg, 225:1-13, 2012.

[18] P. Lin. Theoretical and numerical analysis for the quasi-continuum approximation of a material particle model. Mathematics of computation, 72(242):657-675, 2003.

[19] P.-G. Martinsson and G. J. Rodin. Asymptotic expansions of lattice green's functions. Proceedings of the Royal Society of London A: Mathematical, Physical and Engineering Sciences, 458:2609-2622, 2002.

[20] P.-G. Martinsson and G. J. Rodin. Boundary algebraic equations for lattice problems. Pro- 
ceedings of the Royal Society A: Mathematical, Physical and Engineering Sciences, 465(2108):2489$2503,2009$.

[21] S. F. McCormick. Multigrid methods. SIAM, 1987.

[22] T. Mengesha and Q. Du. Analysis of a scalar peridynamic model with a sign changing kernel. Discrete Contin. Dynam. Systems B, 18:1415-1437, 2013.

[23] W. G. Noid, J.-W. Chu, G. S. Ayton, V. Krishna, S. Izvekov, G. A. Voth, A. Das, and H. C. Andersen. The multiscale coarse-graining method. I. A rigorous bridge between atomistic and coarse-grained models. The Journal of Chemical Physics, 128(24):244114, 2008.

[24] S. Riniker, J. R. Allison, and W. F. van Gunsteren. On developing coarse-grained models for biomolecular simulation: a review. Physical Chemistry Chemical Physics, 14(36):12423, 2012.

[25] S. Silling. Reformulation of elasticity theory for discontinuities and long-range forces. Journal of the Mechanics and Physics of Solids, 48(1):175-209, 2000.

[26] S. Silling and R. B. Lehoucq. Statistical coarse-graining of molecular dynamics into peridynamics. Technical report, Sandia National Laboratories SAND2007-6410, 2007.

[27] S. Silling, R. B. Lehoucq, and M. L. Parks. Connecting length scales with peridynamic mechanics. In Presentation slides from the 2009 Joint ASCE-ASME-SES Conference on Mechanics and Materials, 2009.

[28] S. A. Silling. A coarsening method for linear peridynamics. International Journal for Multiscale Computational Engineering, 9(6), 2011.

[29] S. A. Silling. Origin and effect of nonlocality in a composite. Journal of Mechanics of Materials and Structures, 9(2):245-258, 2014.

[30] E. B. Tadmor, M. Ortiz, and R. Phillips. Quasicontinuum analysis of defects in solids. Philosophical magazine A, 73(6):1529-1563, 1996.

[31] Y. Tao, X. Tian, and Q. Du. Nonlocal diffusion and peridynamic models with Neumann type constraints and their numerical approximations. Appl. Math. Comput., 305:282-298, 2017.

[32] X. Tian and Q. Du. Analysis and comparison of different approximations to nonlocal diffusion and linear peridynamic equations. SIAM Journal on Numerical Analysis, 51(6):3458-3482, 2013.

[33] W. E and P. Ming. Analysis of the local quasicontinuum method. Frontiers and Prospects of Contemporary Applied Mathematics, 6:18, 2005.

[34] X. Wu and X. Li. Simulations of micron-scale fracture using atomistic-based boundary element method. Modelling and Simulation in Materials Science and Engineering, 25(8):085008, 2017. 